\title{
Agent Based Simulation of Group Emotions Evolution and Strategy Intervention in Extreme Events
}

\author{
Bo Li, Duoyong Sun, Shuquan Guo, and Zihan Lin \\ College of Information System and Management, National University of Defense Technology, Changsha 410072, China \\ Correspondence should be addressed to Bo Li; libonudt@gmail.com
}

Received 5 November 2014; Accepted 4 December 2014; Published 28 December 2014

Academic Editor: Francisco Solis

Copyright ( 2014 Bo Li et al. This is an open access article distributed under the Creative Commons Attribution License, which permits unrestricted use, distribution, and reproduction in any medium, provided the original work is properly cited.

\begin{abstract}
Agent based simulation method has become a prominent approach in computational modeling and analysis of public emergency management in social science research. The group emotions evolution, information diffusion, and collective behavior selection make extreme incidents studies a complex system problem, which requires new methods for incidents management and strategy evaluation. This paper studies the group emotion evolution and intervention strategy effectiveness using agent based simulation method. By employing a computational experimentation methodology, we construct the group emotion evolution as a complex system and test the effects of three strategies. In addition, the events-chain model is proposed to model the accumulation influence of the temporal successive events. Each strategy is examined through three simulation experiments, including two make-up scenarios and a real case study. We show how various strategies could impact the group emotion evolution in terms of the complex emergence and emotion accumulation influence in extreme events. This paper also provides an effective method of how to use agent-based simulation for the study of complex collective behavior evolution problem in extreme incidents, emergency, and security study domains.
\end{abstract}

\section{Introduction}

Understanding how the group emotion develops in extreme events is a critical issue in emergency management. Public emergency always occurs with rumors propagating, malicious incitement, and emotion infection, which cause group emotion changing or even extreme behavior $[1,2]$. As a public security incident, extreme group incident is different from general mass incidents in the information propagation manners [3], in which the violence activity information is spreading on the relation network in a relatively private way, such as in the Urumqi incident (Xinjiang, China, 2009) [4]. It is difficult to obtain realistic investigation data of the extreme incidents for the government or researchers due to the privacy and interaction covertness, as well as the complexity of the individual interaction. On the other hand, the group behavior and extreme emotion are hard to detect, which makes it difficult for the strategy formulation and evaluation. Thus, the covert information spreading and related group emotion evolution modeling and analysis become important for incident process understanding and intervention strategy evaluation.

Empirical and theoretical methods both have been used to study the collective behavior evolution process in group incidents [5-9]. The results of empirical studies are different according to each specific event, but they are useful for finding inherent evolution pattern in the incidents $[6,9]$. Theoretical studies try to analyze the collective behavior with mathematical methods, and it is effective in understanding the evolution process from macro perspective [10]. However, both methods have limitations on modeling the complicated system emergence produced by the individual interaction. Agent based Simulation/Modeling (ABS/ABM) provides an effective way to understand the complicated system evolution problem $[11,12]$. It includes models of behavior which observe the collective effects of agent behaviors and interactions [13]. As a simulation method, ABS is usually used along with other methods according to the problem, such as social network $[14,15]$ and system dynamics [16]. The autonomous agents can be used with the heterogeneity and network structure for 
researching the diversity of the agent behaviors, as well as the system complexity from the micro level. Social network is widely used to describe the relation structure between the agents, and the "network agents" is an effective tool to study the collective behavior evolution of agents with relation network [17]. Based on the ABS method, strategies evaluation with computational models is widely used for strategy effectiveness analysis [18]. One of the advantages is that this method provides a way to analyze the strategy effectiveness with repeated experiments, which is impossible for most strategies in real world. Simultaneously, the influence factor analysis can be carried out to understand inherent process of evolution.

In this paper, we construct the ABS models for studying the group emotions evolution process and examine the impacts of three strategies used in incidents intervention: speakers controlling, interaction probability, and communication intervention. The paper is organized as follows. Problem description and research framework are given in Section 2. It is described as mathematical forms, and the research framework gives an overview of this paper. The models used in this research are given in detail in Section 3, including the relation network model, the eventschain model, the information model, and the agent model. Section 4 gives three intervention strategies, as well as evaluation criteria based on the simulation method. Simulation experiments are performed and the results are shown in Section 5, including two virtual scenarios and a real case study. Section 6 concludes the paper.

\section{Problem Description and Research Framework}

2.1. Problem Description and Definition. Emotion and recognition constitute a central element of the human repertoire and the study of their functioning is a prerequisite for the understanding of individual and collective behaviors [19]. The computational modeling (such as ABS) provides a powerful tool to study the individual or collective behaviors as a complex system. This technology has been widely used in emergency management, counterterrorism, and national security. This paper focuses on the group extreme incidents, which are caused by human or organization planning. There are some characteristics of this type of events, so we make three reasonable assumptions for the events description and computational experiments.

The first assumption is that the events in the incidents would not occur at the same time. This means that the events are discrete in the simulation experiments. Although the emotion evolution process may last for a period of time, the events (including related behaviors, such as attributes changings) are independent.

The second assumption is that, at any given moment, all actors act conditionally independently of each other. Such an assumption was already proposed in many studies [20] as a basis for ABS research.

The third assumption is that the strategies are performed completely and ideally. Although the strategy definition and construction depend on the real possibility and feasibility, we assume that there is no case of failure in the experiment.

The general principle of these assumptions is to model the process in a formal framework. Based on the assumptions, we can treat the problem as a behavior evolution process on the network. The individuals can be represented as the nodes of the network and the individual actions are the agent behaviors based on the relation of the network.

The underlying network structure is the basic structure of the diffusion processes which consisted of agents (individuals) and relationships between them. We use the definition of dynamic network in [20]. The set $N$ is a set of social actors (individuals or agents), the relation on $N$ is defined mathematically as a subset of the $N \times N$, and the relation is represented by the $n \times n$ adjacency matrix $X=\left(X_{i j}\right)$. The $X_{i j}=\{0,1\}$, respectively, represents that there is no link or there is a tie between agents $i$ and $j(i, j=1, \ldots, n)$. The agent attributes are assumed to be discrete with a preset interval of values, and $Z_{h i}$ denotes the value of agent $i$ on the $h$ th attribute. The time dependence is indicated by denoting $Z_{h}=$ $Z_{h}(t)$, where $t$ denotes time and $Z_{h}$ is the column containing the $Z_{h i}$ values.

This paper presents models and methods for studying the dynamic evolution process of $\left(Z_{1}(t), \ldots, Z_{H}(t)\right)$. Virtually, the adjacency of the relation matrix $X$ should be included in the dynamic analysis as well, but we here assume that the network remained changeless in a short period (e.g., an event), which is proposed by previous research [21]. In contrast to the individual attributes $Z_{h}$, we are more interested in the collective state, for example, the statistics result of the agents' attributes, which describe the characteristics of the whole collective behaviors and states, and they are also called covariates [20].

Suppose that the observations on $\left(Z_{1}(t), \ldots, Z_{H}(t)\right)$ are available for discrete observation time $t_{1}<t_{2}<$ $\cdots<t_{M}$. The collective attributes could be represented as $\left(F_{1}(t), \ldots, F_{L}(t)\right)$ and $F_{l}(t)=f_{l}\left(Z_{1}(t), \ldots, Z_{H}(t)\right)$, in which $1 \leq l \leq L$. For simplifying, the processes $\left(Z_{1}(t), \ldots, Z_{H}(t)\right)$ and $\left(F_{1}(t), \ldots, F_{L}(t)\right)$ could be represented by the symbol $Y(t)$, and the totality of available data is represented by $y\left(t_{1}\right), \ldots, y\left(t_{M}\right)$.

The interventions strategies are defined as a series of functions acting on the agents set $N$, the relation set $X$, and the attributes set $Z$. We use the symbol $S$ to represent the strategy set, and $S=\left(S_{1}, \ldots, S_{K}\right)$, where the constant $K$ is the number of the strategies. For each strategy, there is a formulation of $S_{k}=f_{k}\left(N, X, Z_{1}(t), \ldots, Z_{H}(t), t\right)$. The $t$ in the formulation means the time on which the strategy performs.

Based on the definition, we could now describe the problem as follows: the initial data include the agents set $N$, the relation set $X$, the attributes matrix of the agents $\left(Z_{1}\left(t_{1}\right), \ldots, Z_{H}\left(t_{1}\right)\right)$, and the covariates set $\left(F_{1}\left(t_{1}\right), \ldots\right.$, $\left.F_{L}\left(t_{1}\right)\right)$; the agents will take actions (interaction) during each time step, which makes the values of $Z$ and $F$ changing; the purpose of this paper is to understand the dynamic evolution process of the collective emotions $F$ and to observe the effectiveness of different intervention strategies in the evolution process. 


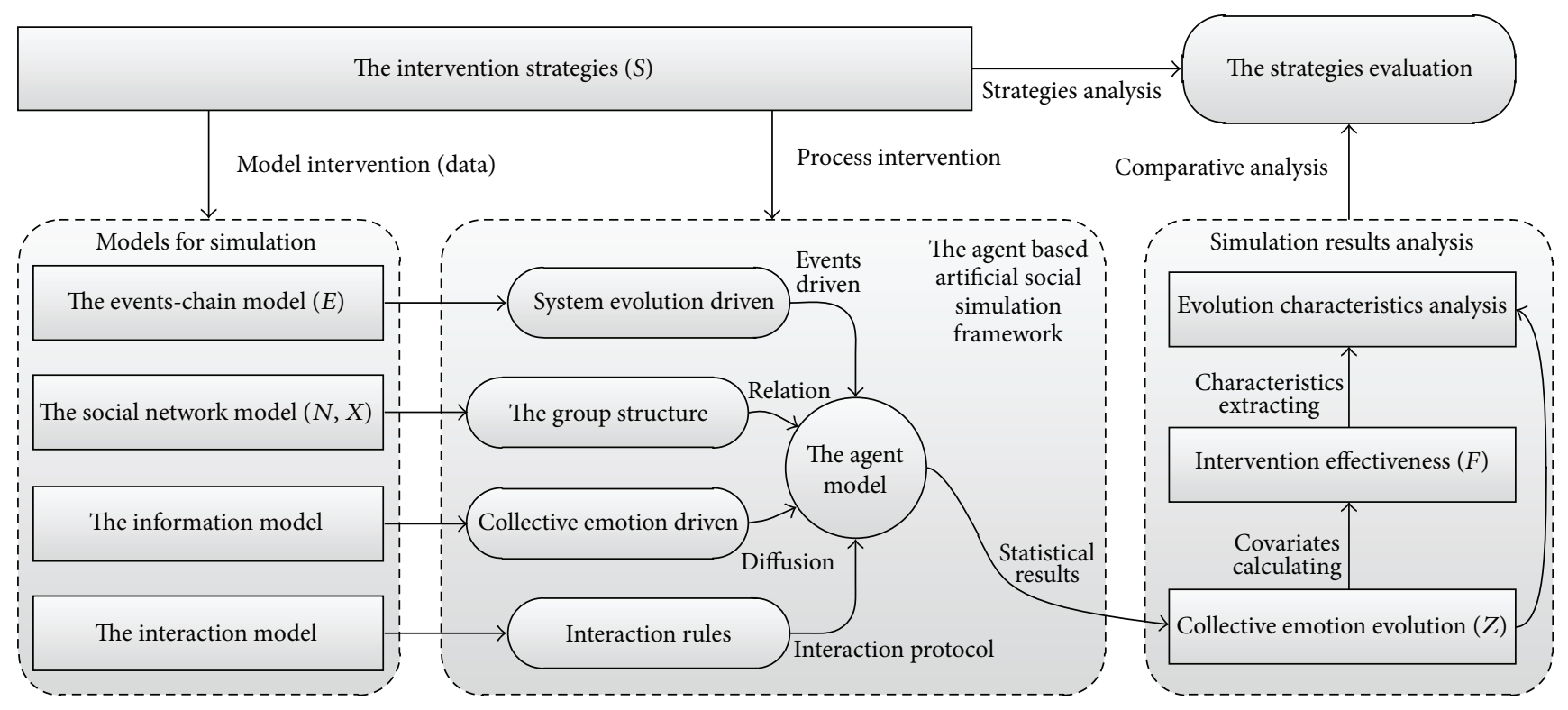

Figure 1: The research framework of collective emotion simulation and strategy evaluation.

2.2. Research Framework. Based on the problem definition mentioned above, the research framework can be constructed for the sake of emotion evolution process analysis and intervention strategy evaluation. As shown in Figure 1, this work consists of the following four components. (1) The agent based artificial social simulation framework, involving frequent interactions between individuals within the group and the resulting changes of collective emotion state during the extreme emotion diffusion. (2) Models for simulation, including four models used in the simulation experiments, the events-chain model as system evolution driven, the social network model as the group structure (agent interaction environment), the information model as the collective emotion driven, and the interaction model as the interaction rules in the agent based simulation (ABS) method. (3) Simulation experiments results analysis, providing the statistical results of the collective emotion evolution $(Z)$, the covariates results for the intervention effectiveness $(F)$, and the characteristics analysis of the evolution process. (4) The intervention strategies and evaluation, including the strategies construction with mathematical forms and the effectiveness evaluation.

The core module of the method is the ABS structure, which is used to model the complex interaction behavior between the individuals and to observe the collective emotion emergence. The models for simulation are built to describe the specific environment and behavior in the corresponding scenarios of the extreme events, such as the successive terrorism events, the information diffusion process, and the individual interaction. With simulation time going on, the statistical results are changing with the individuals' behaviors, and the collective emotion criteria capture the evolution dynamics from the macro perspective. Thus the simulation results analysis is a dynamic process describing the macro collective emotion state. The strategies effectiveness is analyzed by the covariates calculating and the evolution characteristics analysis, which provides results for strategies evaluation. The strategies make influence in two ways, the model intervention (data aspect) and the process intervention (interaction aspect).

\section{Model Description}

3.1. The Relationship Network Model. In extreme events, for covertness, information spreading is always invisible and acts in relatively covert ways instead of public channels, such as email, instant messaging, or even mouth-to-mouth way. During the process, the personal relation plays an important role in the communication object selection. In this method, the BA network model [22] is used as the relation structure, which can be seen as the interaction environment rather than the behavior model, given it can format the rules of agent selection in interaction. During the period of an event, the network structure can be treated as a static state [21].

3.2. The Events-Chain Model. In order to simulate the temporal successive events, two structures are proposed, the "events-chain" structure and "meta-event" structure. The "meta-event" structure is a unit of single event or can be called episode, which is the component unit of the events-chain. For example, it can be rumors spreading process or an action of an organization. It also includes the following related group response activities. The "events-chain" structure consists of series of temporal "meta-events," and the structure makes it capable of describing the complex event process for the simulation. The accumulated emotion of events is expressed through the agent model, which makes it possible to analyze the events related collective emotion and behavior evolution in a computational experimentation way.

Figure 2 illustrates the events-chain model of temporal series events. In the model, the nodes represent the metaevents, which are ordered by the temporal relations and 


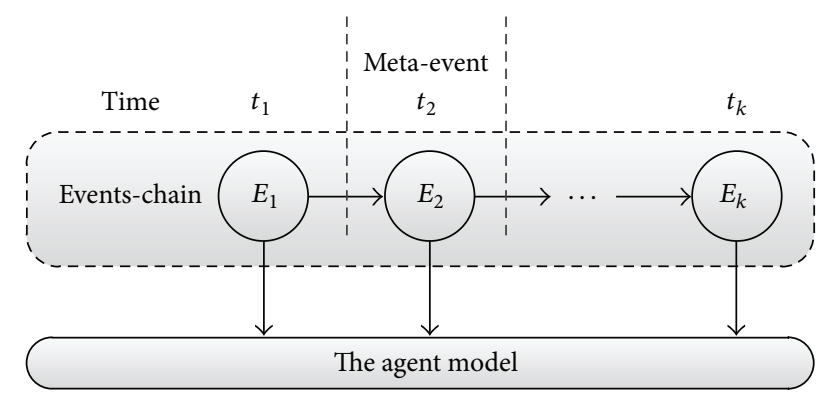

FIGURE 2: The events-chain model of temporal successive events.

denoted as $E_{k}$. The attributes of the event can be noted as $E_{k l}$, which includes the event criticality $\left(E_{k 1}\right)$ and the start time $\left(E_{k 2}\right)$. The criticality represents the event "importance" level to the individuals. When the criticality value is high, the group response will be violent, and when it is low, the collective behavior will be peaceful. Thus the successive events can be modeled as a set $E=\left\{E_{1}, E_{2}, \ldots, E_{K}\right\}$ and the criticality set $I=\left\{E_{11}, E_{21}, \ldots, E_{K 1}\right\}$ and start time set $T=\left\{E_{12}, E_{22}, \ldots, E_{K 2}\right\}$. The accumulated effects of events militate through the agent attributes and behavior, which will be described in the agent model.

3.3. The Information Model. The events usually occur accompanying event information in the forms of news, rumors, or extreme activity information. Information transferring between agents makes agent attributes and behavior changing. The information spreading process of meta-event is similar to the rumor spreading process, but the difference is that the impact can be accumulated during the period of event influence time. According to the event information set, each agent has an event information set of $Z I_{i}=\left\{Z E_{1}, Z E_{2}\right.$, $\left.\ldots, Z E_{K}\right\}$, and $Z E_{k}=\{0,1\}$.

We assume that, after the individual receives the information, it will be transmitted, which is different from the classic rumor propagation model [5]. Agent will randomly select another agent who connects with it to send the message and exchange opinion, rather than treating the information as several statuses. This is because we have found that the extremists or radicals will send the violent activity information over and over again even though the object has got the information [4].

3.4. The Agent Model. The agent model describes the attributes and behavior rules in the simulation. As mentioned before, the attributes are the values of $Z$, and the agent behavior can be modeled as a series of functions on $Z$.

\subsubsection{Agent Attributes}

Autonomy. Autonomy is an important variable when considering the information propagation in agent based simulation [23]. The autonomy of an agent will influence the acceptable level of the external information, which expresses the impact on the behavior of the individual [8]. It determines the extreme emotion level after the individual receives the information and the impact of environment by the other individuals, denoted as $Z_{1}$.

Extreme Emotion. There are many researches on the emotion model and emotion agent $[24,25]$; however, it is difficult to build a uniform model to describe the emotion changing process due to the complexity of emotion and its influence factors. Based on the KISS rule (keep it simple and stupid) [26], we define three variables to model the extreme emotion in this specific application scenario, the individual selfgenerated emotion $Z_{2}$, environmental influence emotion $Z_{3}$, and individual extreme emotion $Z_{4}$. Self-generated emotion describes the individual's own level of radicalization, and the environmental influence emotion describes the conformity degree of an agent to the others.

Emotion Decay Rate. For individuals there is a process of mood self-adjustment, so extreme emotion has a decay process. If there is no external information within a period of time, individual mood will gradually calm down. This is another factor that affects the extreme emotion evolution and is denoted as $Z_{5}$. Meanwhile, after receiving the information for a certain period of time, the emotions are hardly affected by other external individuals, which means $Z_{3}$ will be zero after a time threshold, labeled as $\sigma$.

\subsubsection{Personal Interaction Model}

Self-Generated Emotion. After an agent receives the event information, the self-generated emotion will be generated by the agent itself, and it can be modeled as a function of the individual autonomy and the event criticality $E_{k 1}$, which can be shown as follows:

$$
Z_{2 i}=f\left(Z_{1 i}, E_{k 1}\right)=Z_{1 i} \times E_{k 1}
$$

Actually, the individual self-generated emotion is related with many factors, such as the content of events, individuality, and other related social attributes. These factors involve more complex questions and event classification issues of individual emotional tendencies, which are out of scope of this paper. Considering the simulation scenario, we use a multiplication rule.

Environmental Emotion. The extreme emotion contagion process occurs among the individuals through the relation network; thus, the emotion of agent will be affected by the others connecting with it. Assume the number of the agents who connect with agent $i$ and have received the event information is $d$; then, the environmental emotion of an agent can be expressed as

$$
Z_{3 i}=\frac{\left(\sum_{X_{i j}=1} Z_{4 j}\right)}{d},
$$

where $i \neq j, 1 \leq i, j \leq n$; thus, the agent extreme emotion can be calculated as follows:

$$
Z_{4 i}=Z_{1 i} \times Z_{2 i}+\left(1-Z_{1 i}\right) \times Z_{3 i}
$$


where the $Z_{1 i}$ is used as a weight between self-generated emotion and environmental emotion. The extreme emotion of agent $i$ on the event $k$ at time $t$ can be represented as $Z_{4 i}^{k}(t)$.

Extreme Emotion Evolution. Individual extreme emotion is a process of accumulation, and it can reach a high value enough for people to take extreme actions. On the other hand, the extreme emotion has a period of decay. As suggested in [25], the emotion decreases with the time, but it does not go away instantaneously, so the decay process is represented via an exponential function. We assume that only the self-generated emotion decays, and the environmental emotion depends on the emotions of the connected agents. Given the initial emotion $Z_{2 i}\left(t_{0}\right)$, the current time $t$, and the decay rate $Z_{5 i}$, the decay function is given by

$$
Z_{2 i}(t)=Z_{2 i}\left(t_{0}\right) \times e^{-Z_{5 i} \times\left(t-t_{0}\right)} .
$$

The accumulation effect of the extreme emotion comes from the successive events influence. For each event, every agent has an event emotion according to the information. At time $t$, the extreme emotion of agent $i$ should be calculated as follows:

$$
Z_{6 i}(t)=\sum_{k=1}^{K} Z_{4 i}^{k}(t)
$$

\section{Intervention Strategies}

The purpose of this study is to understand the evolution process of the collective emotion. In addition, we are more interested in how to intervene in the extreme emotion spreading. We call the intervention method used in the spreading process as intervention strategies in this paper. Valente surveys four strategies on network intervention, which are individuals, segmentation, induction, and alteration [27]. Based on the formal description mentioned before, we give the details of the three strategies examined in this method.

4.1. Speakers Controlling. Speakers controlling strategy involves eliminating speakers of the network who are the source of the events information, which may also be taken as leader-focused strategy [18]. The speakers here refer to the people that spread the information and emotion at the first time, such as the schemer of the event, the leader of the group, and a member of the terrorist organization. This strategy is commonly referred to as capturing targets, just as key players in social network mentioned in [28]. The motivation of this strategy is to intervene in event information spreading and emotion diffusion from the source, so as to control or prevent the event occurrence, with the understanding that eliminating the speakers in the network should have the heaviest influence on the collective extreme emotion evolution process.

Formally, we note speakers controlling strategy as $S_{1}$; thus, removing the speakers could be treated as a function transformation from the node set $N$ and the link $X$ to the sets $N_{1}$ and $X_{1}$. Let $N_{1}^{\prime}$ denote the speakers set, so $N_{1}=$ $N-N_{1}^{\prime}$. Here we use $X_{1}^{\prime}$ to denote the link subset of $X$ which includes all the links related with the agents in $N_{1}^{\prime}$; that is, $X_{1}^{\prime}=\left\{X_{i} \mid i \in N_{1}^{\prime}\right\}$, and $X_{1}=X-X_{1}^{\prime}$. In addition, the agents in $N_{1}^{\prime}$ will be inaccessible for any other agent once they are removed.

4.2. Interaction Probability. The emotion contagion needs continuous interaction between the individuals [29]. This strategy is based on the understanding that if the agents interact with lower probability, the extreme emotion would generate slower, and less people would be affected. This strategy usually associates with event popularity, and high popularity will make people exchange the opinion and information more frequently. Empirical studies based on online social media have provided the evidence of topics intervention [30], which is known as public opinion guidance.

The probability in this strategy means the contact frequency in a fixed time period, and its interval is $[0,1]$. We use $P_{\text {in }}$ to represent the interaction probability between each pair of agents. Generally it should be a probability distribution, and the probability is generated in each time step. We use $S_{2}$ to represent this strategy; thus, $S_{2}$ is a probability matrix of the link set $E$, which generates the link active probabilities in each time step corresponding to the probability distribution. To simplify the simulation, it is used as a given value in this method.

4.3. Communication Intervention. In contrast to interaction probability strategy, communication intervention strategy takes actions of deleting the interaction pathways between agents, with the idea that fewer links will mitigate the information diffusion and emotion cognation process [31]. The communication in this strategy refers to the ways that agents keep in touch with each other, such as telephone, email, instant messenger, and website. The motivation of this strategy is to reduce the communication channels between individuals, so as to intervene in the process of emotion evolution and even the collective behavior.

This strategy is different from the two previous strategies from the structural perspective. Firstly, the communication strategy interdicts the links between the agents which are still in the network, and the speaker control strategy removes the agents and the related links of the agents; secondly, all the agents' links may be affected by the communication strategy and only speakers' links in the speaker strategy; thirdly, the interaction probability strategy reduces the frequency of interaction, and the links still exist in the network, which would be removed in communication strategy. This method is not usually taken, because it needs to interrupt the daily communication device and related network, which may involve the security department.

This strategy is denoted as $S_{3}$. Consequently, removing the links could be seen as a transformation of $X \rightarrow X_{3}$. We assume the obstructed link set is $X_{3}^{\prime}$, so $X_{3}=X-X_{3}^{\prime}$. The key problem is how to get the link set $X_{3}^{\prime}$, that is, how to determine the links to be removed. As the communication strategy is not a target-focused method, here we use a probability $P_{\text {re }}$ to represent the probability of each link to be removed. 
4.4. Strategy Effectiveness Evaluation. To evaluate effectiveness of the strategies, the criteria should be proposed. The basic principle of artificial society simulation is to observe the complex emergence results of simple agent interaction [32], and one of the effective approaches is to evaluate the statistical characteristics of the group attributes. As mentioned above, the effectiveness evaluation should be a series of observations with the time, using the notation $\left(F_{1}(t), \ldots, F_{L}(t)\right)$. Besides the statistical variables, the characteristics of the statistical results curves are also important to evaluate the effectiveness of the strategies. For distinction analysis, the characteristics of the dynamic results curves are noted as $\left(T_{1}, \ldots, T_{M}\right)$. In this study, nine statistical criteria are used as follows.

(i) The maximum number of extreme group agents during event $\left(F_{1}\right)$. To specify the agents' emotion level, the distinction threshold is used to determine whether the agent belongs to the group. Specifically, in our model, the extreme emotion needs to reach a certain threshold $\tau_{1}$ at which the agent can be seen with "extreme emotion."

(ii) The maximum number of agitated group agents during event $\left(F_{2}\right)$. The distinction method of agitated agents is similar to the extreme group with the threshold $\tau_{2}$. The agent that belongs to this group is between the states "extreme" and "gentle," who may be incited to the state "extreme" or calm down to the state "gentle."

(iii) The maximum value of average emotion during event $\left(F_{3}\right)$. The average emotion is a measurement of the total group emotion state, and it is also a symbol of the collective behavior.

(iv) The duration of event (the time from the event starting to the ending, denoted as $T_{1}$ ).

(v) The duration when average emotion value is above a threshold $\left(\tau_{3}\right)$. This duration can be seen as the "risk period" of the incident $\left(T_{2}\right)$.

(vi) The length of time between the event starting and the time when the number of extreme group agents reaches the peak value $\left(T_{3}\right)$.

(vii) The length of time between the time when the number of extreme group agents reaches the peak value and event ending $\left(T_{4}\right)$.

(viii) The length of time between the event starting and the time when the number of agitated group agents reaches the peak value $\left(T_{5}\right)$.

(ix) The length of time between the time when the number of agitated group agents reaches the peak value and event ending $\left(T_{6}\right)$.

\section{Simulation Experiments and Results Analysis}

In the previous sections, we have specified the mathematical description of the problem, the research framework of this study, the emotional dynamics of agents, the models used for simulation, and the intervention strategies with nine specific criteria for effectiveness evaluation. To numerically investigate the method proposed, we need to specify the setup in terms of the network, the initial conditions of agents, and the parameters for experiments, which are all described in this section. Simultaneously, the simulation experiments are given as well as the results analysis and validation, including a real case study.

5.1. Simulation Scenarios Setting. The procedures of the simulation experiments are as follows.

(1) The initial social network was generated by BA network model as the local relation structure of agents.

(2) The simulation step was 200 steps for single event, and 250 for two correlated events. The time step was updated as follows: as the system time increases, the time step is updated, and each agent selects one of the agents to interact.

(3) At the time the event occurred, the event information was given to a designated start node (speaker). The default node was the node with maximum degree in the network.

(4) At each time step after the incident, the agent's selfgenerated emotion in the event was in attenuation with the decay rate, and the environmental emotion updated based on the other agents' emotions.

(5) When an agent received new information, it would only be affected by the agents who had also received the latest information and no longer affected by the past events.

(6) After an agent received information for a time threshold $(\sigma)$, the agent was no longer affected by the emotions of other agents about the event.

Before starting the simulation experiments, the settings of the initial parameters were fixed at default values (see Table 1).

5.2. Collective Emotion Evolution of Single Events. We first investigated the main effect of different strategies taken on single event. In each run, the strategy was taken once at the same simulation time step (step 25, 5 steps after the event starting). Figure 3 shows the group emotion evolution process under different strategies. The numerical results are presented in Table 2.

As in the figure, the curves of speakers controlling strategy and the curves without strategy are almost overlapping. The results indicate that if the speakers controlling strategy is taken after the event start time, it is almost invalid. This is because the event information has already been propagated, and the extreme emotion of the agents will also be generated without the speakers. This is an interesting result because removing the key nodes is usually considered as a very useful strategy in counterterrorism strategy, and it has little effect on collective emotion. As expected, reducing the interaction probability between the agents not only effectively reduces the number of agents in each group, but also slows the growth of 
TABLE 1: The initial parameters settings found.

\begin{tabular}{|c|c|c|c|c|}
\hline Simulation parameters & Notation & Range & Default & Object of parameter \\
\hline Autonomy & $Z_{1}$ & {$[0,100]$} & Random & Agent (person) \\
\hline Self-generated emotion & $Z_{2}$ & {$[0,500]$} & 0 & Agent (person) \\
\hline Environmental emotion & $Z_{3}$ & {$[0,500]$} & Random & Agent (person) \\
\hline Extreme emotion & $Z_{4}$ & {$[0,500]$} & 0 & Agent (person) \\
\hline Decay rate & $Z_{5}$ & {$[0.05,0.1]$} & Random & Agent (person) \\
\hline Influence time & $\sigma$ & {$[1,100]$} & 50 & Agent (person) \\
\hline Criticality & $E_{k 1}$ & {$[1,5]$ (int) } & Given & Event \\
\hline Events start time & $E_{k 2}$ & - & 20 & Event \\
\hline Agent number & $N$ & - & 10000 & Network/group \\
\hline BA node link number & - & {$[1,100]$} & 6 & Network/group \\
\hline Extreme group threshold & $\tau_{1}$ & {$[0,500]$} & 400 & Agent emotion \\
\hline Agitated group threshold & $\tau_{2}$ & {$[0,500]$} & 200 & Agent emotion \\
\hline Average emotion threshold & $\tau_{3}$ & {$[0,500]$} & 100 & Group average emotion \\
\hline Interaction probability & $P_{\text {in }}$ & {$[0,1]$} & 0.5 & Interaction probability strategy \\
\hline Removing probability & $P_{\text {re }}$ & {$[0,1]$} & 0.3 & Communication intervention strategy \\
\hline Strategy execution time & - & - & 25 & Strategy \\
\hline
\end{tabular}

TABLE 2: Strategy effectiveness results of single event.

\begin{tabular}{lcccc}
\hline Criteria & Without strategy & Speakers controlling & Interaction probability & Communication intervention \\
\hline$F_{1}$ & 541 & $537(0.01 \%)$ & $327(-39.55 \%)$ & $479(-11.46 \%)$ \\
$F_{2}$ & 8223 & $8201(0.00 \%)$ & $6008(-26.94 \%)$ & $7122(-13.39 \%)$ \\
$F_{3}$ & $250.39(0.00 \%)$ & $209.58(-16.48 \%)$ & $230.22(-8.26 \%)$ \\
$T_{1}$ & 122 & $124(+1.64 \%)$ & $158(+29.51 \%)$ & $122(0.00 \%)$ \\
$T_{2}$ & 45 & $45(0.00 \%)$ & $44(-2.22 \%)$ & $45(0.00 \%)$ \\
$T_{3}$ & 16 & $17(+6.25 \%)$ & $29(+81.25 \%)$ & $17(+6.25 \%)$ \\
$T_{4}$ & 40 & $40(0.00 \%)$ & $49(+22.50 \%)$ & $43(+7.50 \%)$ \\
$T_{5}$ & 20 & $20(0.00 \%)$ & $34(+70.00 \%)$ & $21(+5.00 \%)$ \\
$T_{6}$ & 102 & $104(+1.96 \%)$ & $125(+22.55 \%)$ & $102(0.00 \%)$ \\
\hline
\end{tabular}

emotion, as well as the information diffusion process. This may be because the information transfer process between agents has been delayed (Figure $3(\mathrm{~d})$ ). The communication strategy (removing part of the links) reduces the maximum of both groups and the average emotion, but it only slows the information diffusion process to some extent. Both the time period of groups and the average emotion have little difference.

Table 2 presents the numerical results of strategies effectiveness on single event. The values in the parenthesis are the difference rates. As expected, the interaction probability strategy is the most effective method in reducing the number of each group's agents and the average emotion. The speakers controlling strategy makes nearly no difference as the event information has been propagated. The communication intervention strategy will reduce the number of agents and average emotion to some extent. From time aspect, the effect of speakers controlling strategy has no obvious difference, so is the communication strategy. The interaction probability significantly affects the "rise phase" of collective emotion $\left(T_{3}\right.$ and $T_{5}$ ) and the "fall phase" (about 20\%). All the strategies have no obvious effect on the length of "risk period" $\left(T_{2}\right)$.
Furthermore, Figure 4 shows the collective emotion distribution of different strategies at the peak points. As in Figure 4(a), the interaction probability strategy reduces the mean value of the emotion and also the number of agents with high emotion value. The speakers controlling and communication strategies also have little effect. In Figures 4(c) and $4(\mathrm{~d})$, the agents with emotion zero are those who have not received the event information. The self-generated emotion is approximately uniformly distributed (Figure 4(c)), given it is only determined by the autonomy of the agents, the decay rate, and the event criticality. On the contrary, the environmental influence emotion approximately follows a normal distribution (Figure $4(\mathrm{~d})$ ). This is an interesting result, and it indicates that, at the collective emotion peak point, the environmental influence emotion plays a dominant role for an agent to be an extreme agent. Moreover, in the scatter diagram of 2-dimension distribution (Figure 4(b)), more points are above the symmetry line (red line), and this also indicates that the environmental emotion is higher than the self-generated emotion for most agents. This interesting result means that the environmental emotion is the main cause for an agent to become extreme. 


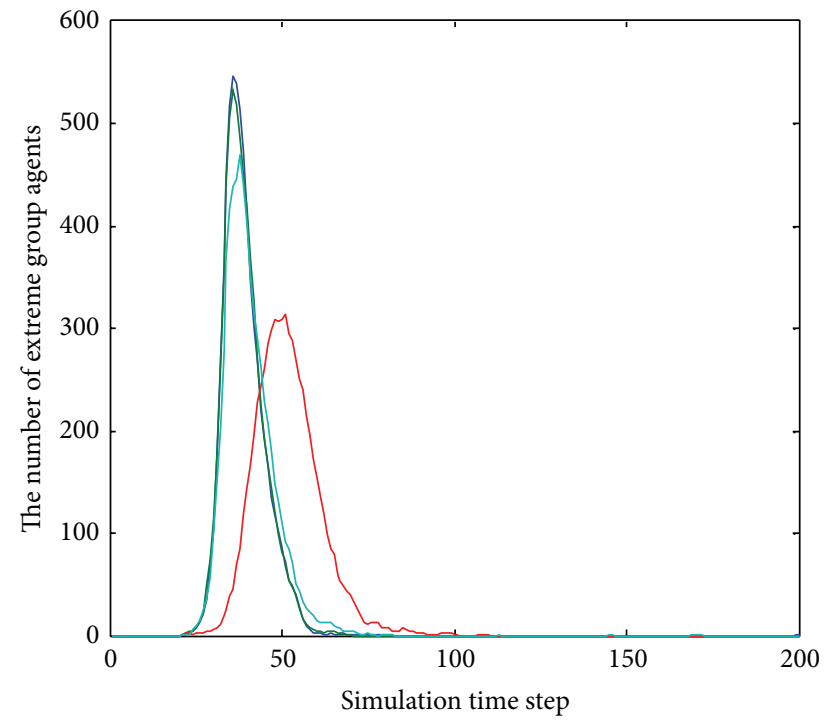

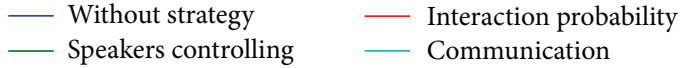

(a)

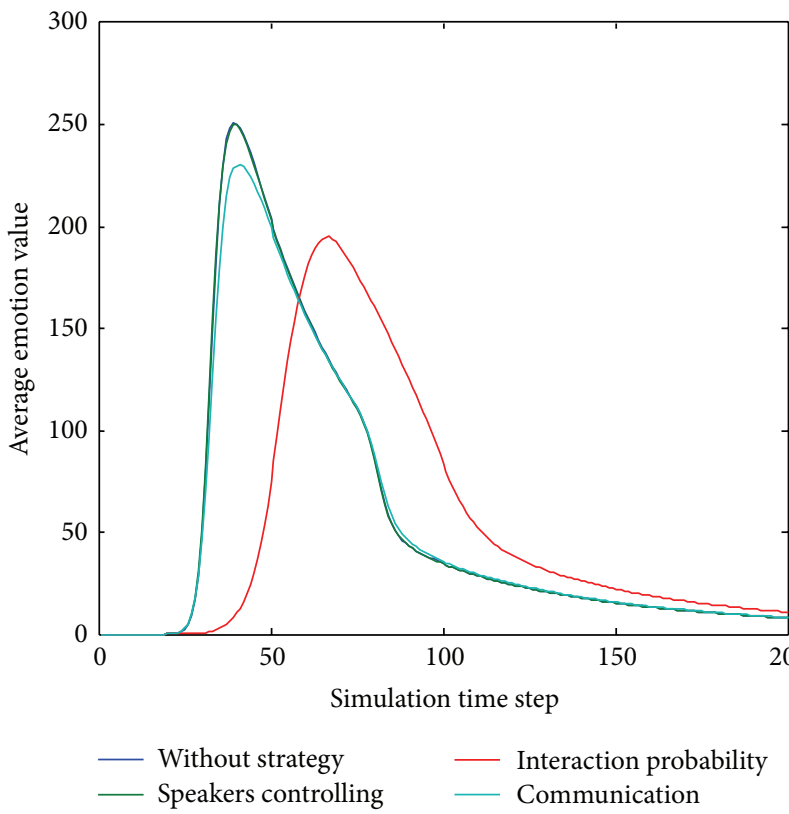

(c)

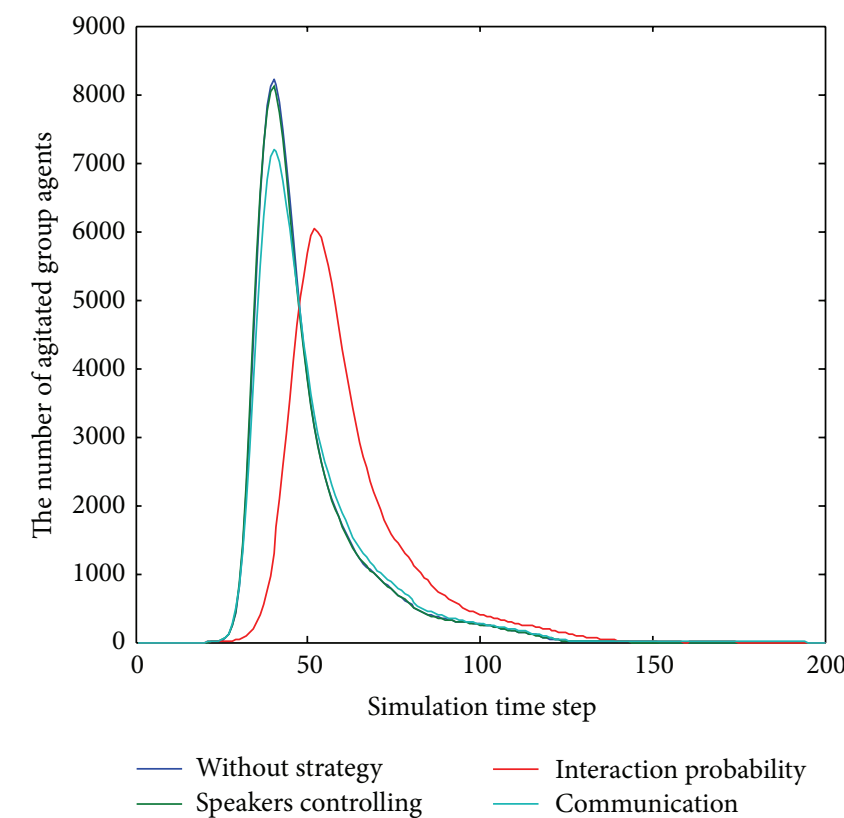

(b)

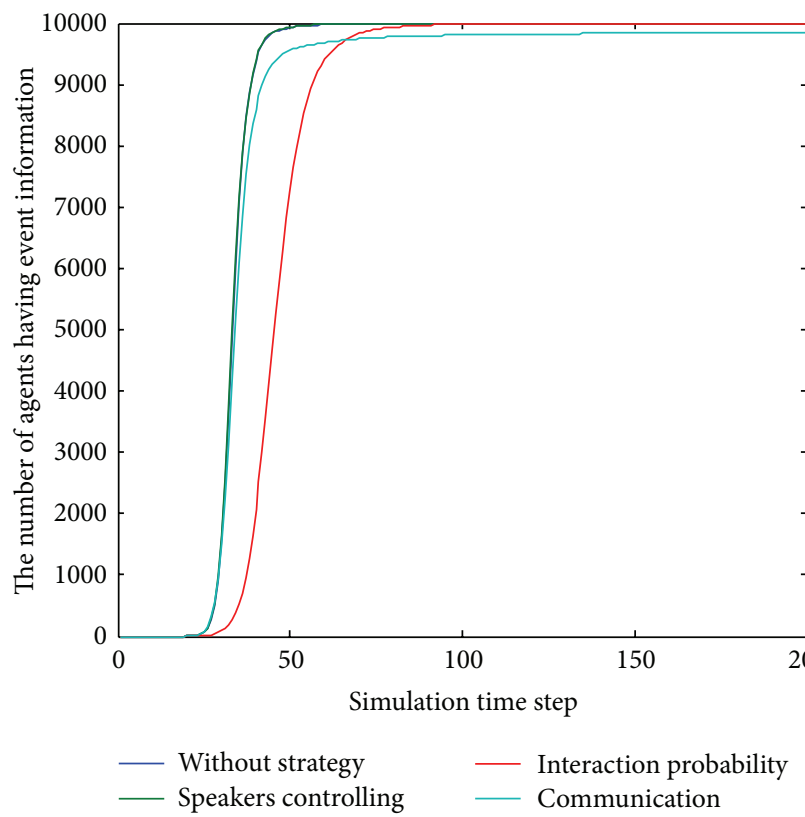

(d)

Figure 3: Collective emotion evolution curves of single event, (a) the number curves of extreme group agents, (b) the number curves of agitated group agents, (c) average emotion value evolution curves, and (d) the information spreading process curves.

5.3. Group Emotion Evolution of Events-Chain Model. We then discuss the results of experiments under different strategies with events-chain model. Because of the accumulation effect, the emotion evolution will be different from that in single event. Figure 5 shows the results of two correlated events under different strategies. The two events start, respectively, at time steps 20 and 60, and both with criticality of 5 . All the other parameters are set as default values in Table 1. The curves of the two events are obviously different from the single event, especially the curves of the second event. The numbers of extreme group agents are much higher in the second event than in the first one (Figure 5(a)). This is due to the emotion accumulation influence, as the start time of the second event is in the influence time of the first event. In contrast, the numbers of the agitated group agents reduce because of the constant total number of agents (Figure 5(b)). According to the results, the speakers controlling strategy is the most effective method to reduce the collective extreme 


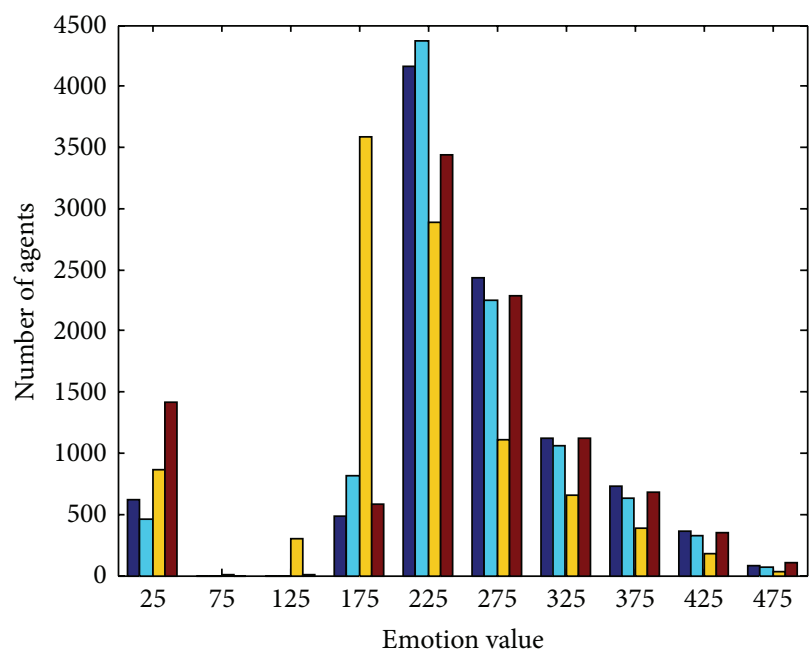

Without strategy $\square$ Interaction probability Speakers controlling Communication

(a)

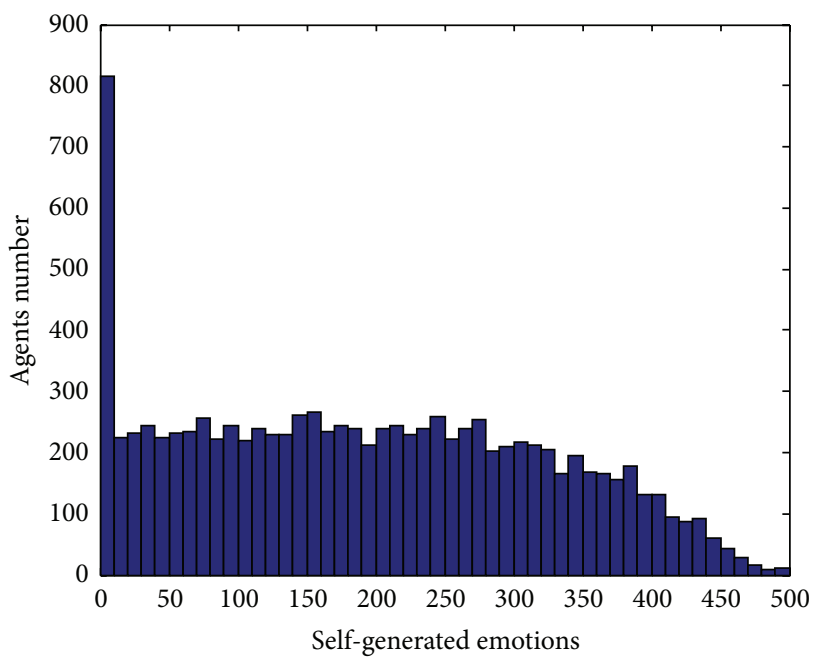

(c)

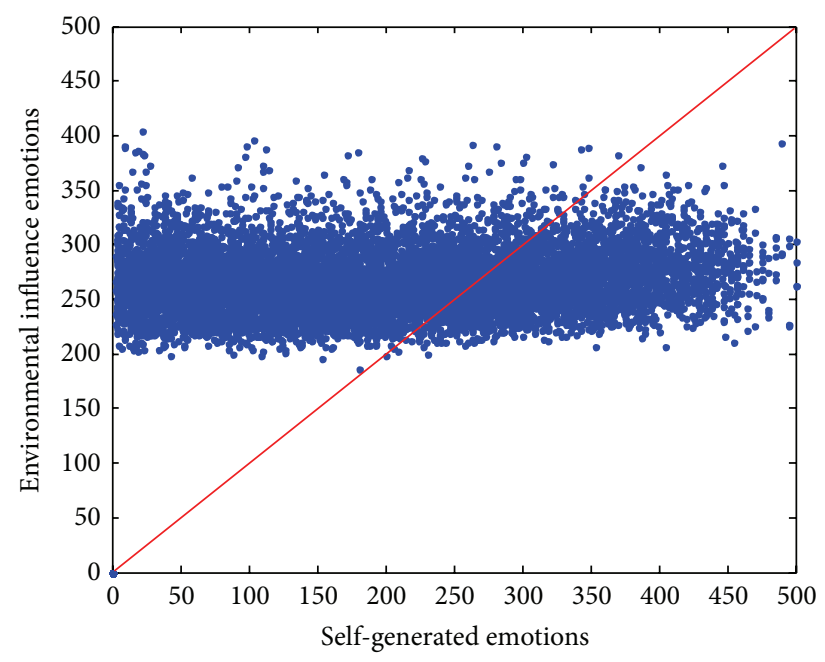

(b)

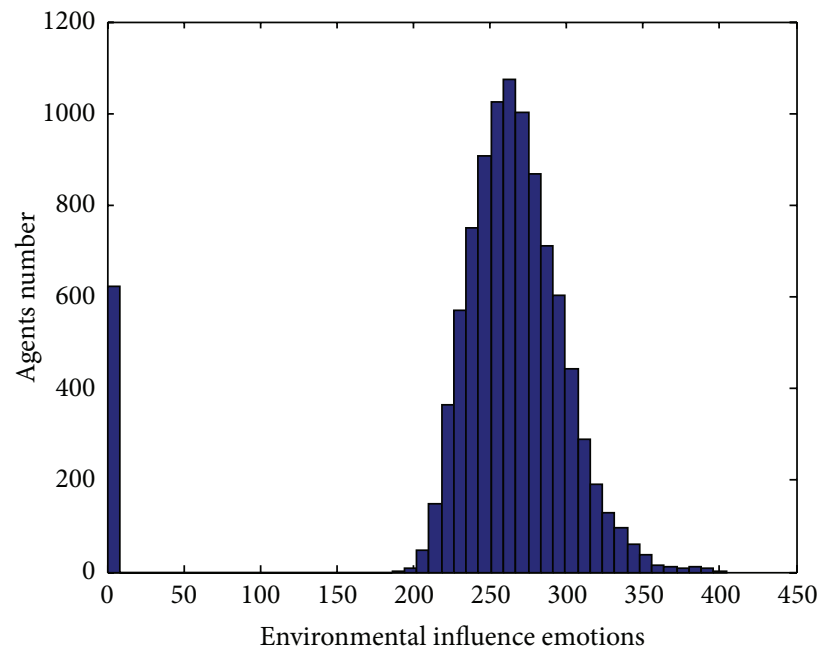

(d)

FIGURE 4: Emotion components distribution at the peak points: (a) emotion distribution of agents in different strategies, (b) scatter diagram of 2-dimension emotion distribution (without strategy), (c) self-generated emotion distribution (without strategy), and (d) environmental emotion distribution (without strategy).

value, because the information of the second event cannot be transferred (Figure 5(d)) since the speakers have been removed from the network.

As in single event, the same nine criteria are used for strategy effectiveness analysis. Unlike the single event, we are more interested in the accumulation influence on the second event. Thus, in this experiment all the time indicators describe the characteristics of the second event (except $T_{2}$ ), and the numerical results are shown in Table 3. The percentage in the column of "without strategy" is calculated based on the single event (column 2), and the others are calculated based on the accumulation events (column 3, without strategy).
Firstly, the difference between the single event and accumulation event is obvious, especially the number of extreme group agents $(+1081.90 \%)$. On the contrary, the number of the agitated group agents decreases due to the constant total agents. Another difference is that the time indicators all increase, especially the "fall phase" of the two groups. It is interesting that although the maximum number of the agitated groups has decreased $\left(F_{2},-24.76 \%\right)$, the time length still increases $\left(T_{6},+48.65 \%\right)$. Secondly, the speakers controlling is the most effective strategy for correlated events; this is because removing the speakers from the network directly prevents the second event from taking place (as analysis of Figure 5). Thirdly, the effectiveness of interaction 


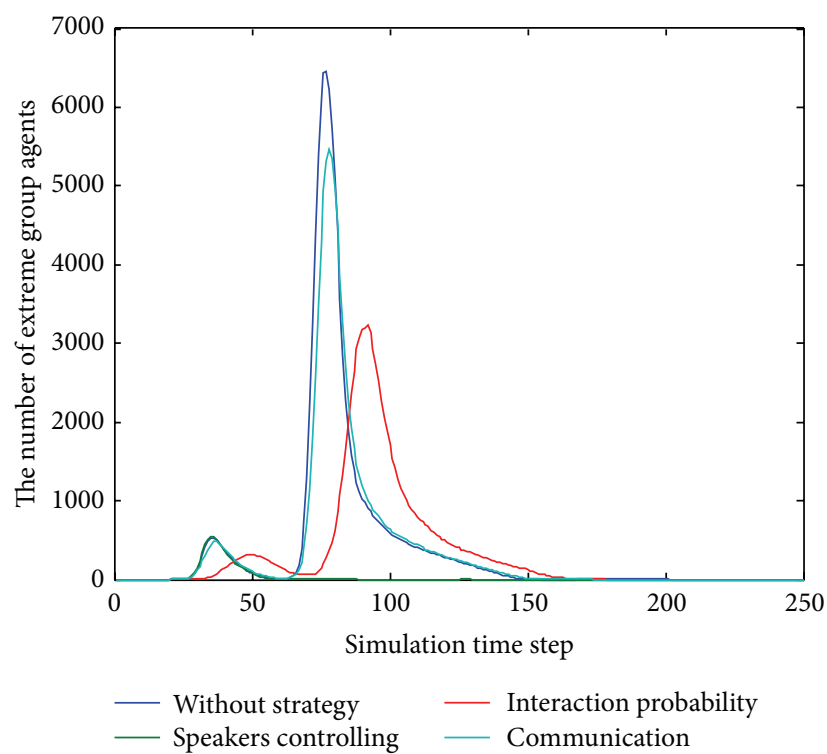

(a)

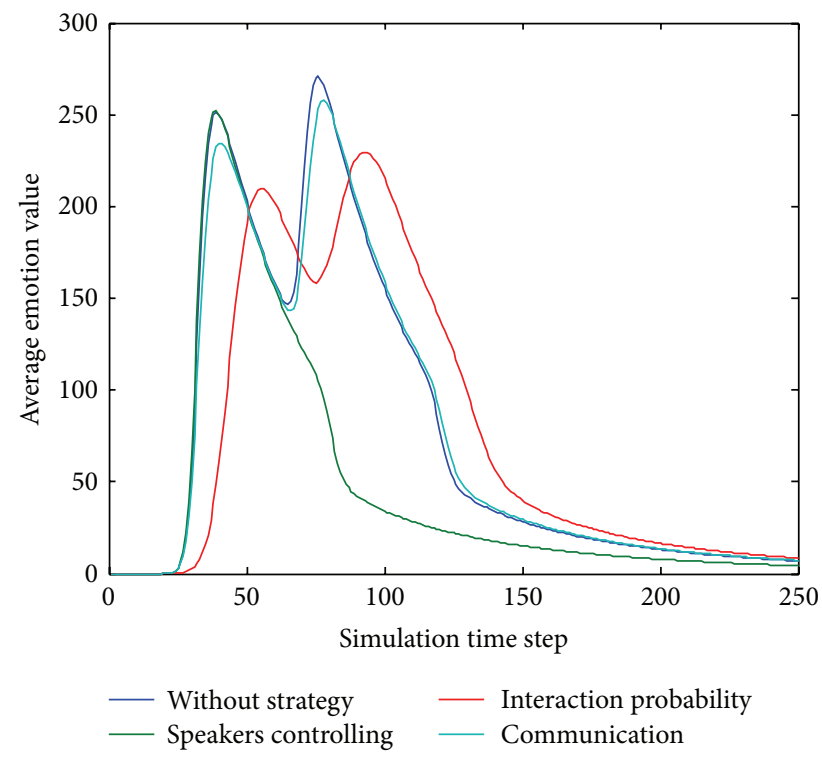

(c)

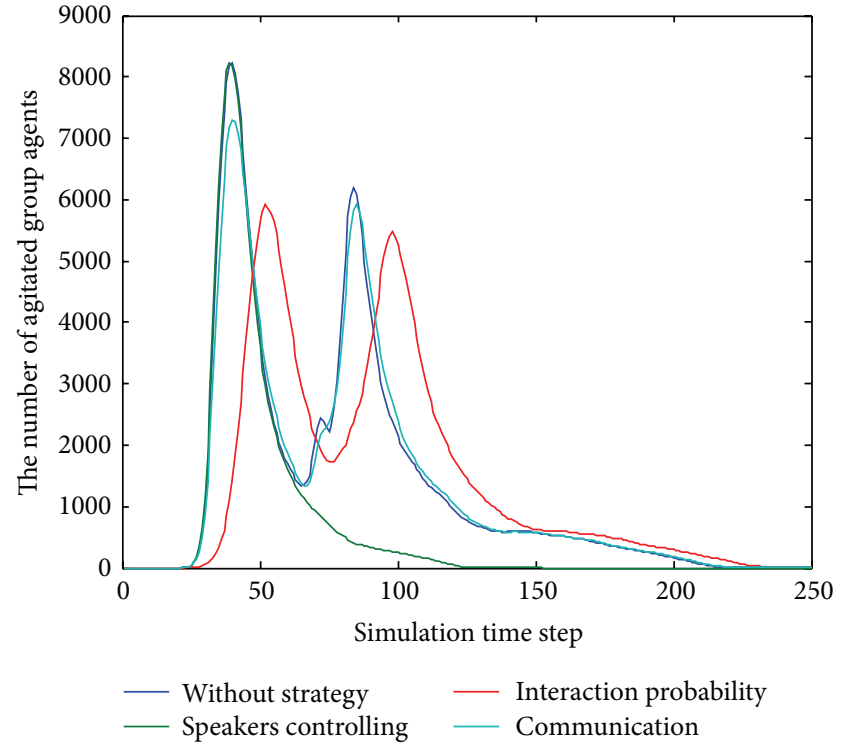

(b)

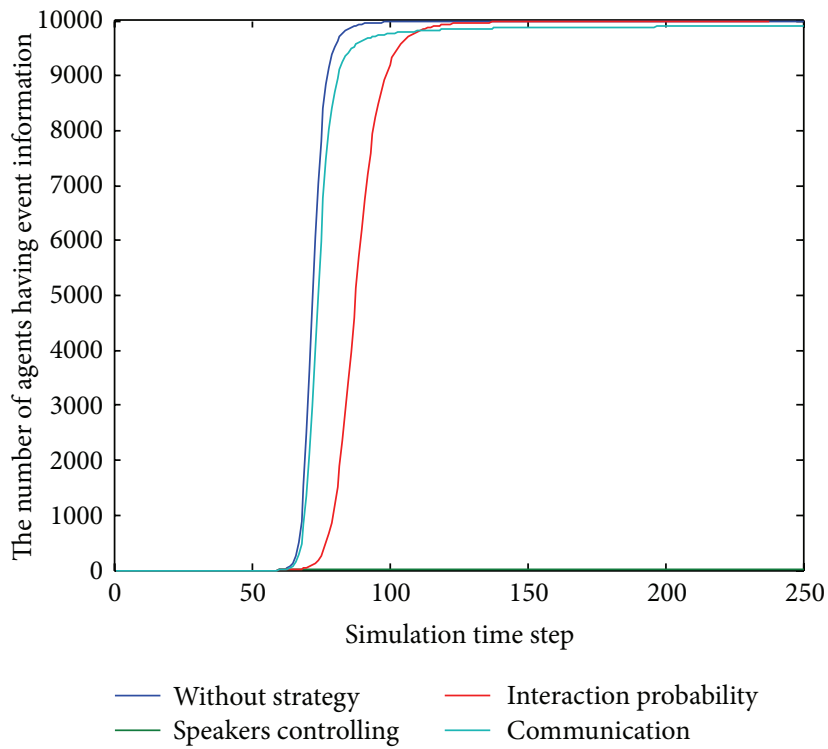

(d)

FIGURE 5: Group emotion evolution curves of successive events: (a) the number of extreme group agents; (b) the number of agitated group agents; (c) average emotion value evolution curves; (d) the information diffusion curves (the second event).

probability strategy on the collective emotion has no obvious difference as in single event, but the time indicators change a lot. The duration of event shortens a little $\left(T_{1},-1.57 \%\right)$, as well as the "fall phase" of the two groups (resp., $-30.65 \%$ of extreme group and $-9.70 \%$ of agitated group). Lastly, the effectiveness of communication strategy becomes weaker, such as the duration of the event time $\left(T_{1}\right)$, the "fall phase" of the extreme and the agitated groups $\left(T_{4}\right.$ and $\left.T_{6}\right)$. In sum, accumulation influence makes the collective emotion stronger, and it will weaken the strategy effectiveness except the speakers controlling strategy.
5.4. Model Validation. Validation is a critical issue for any modeling approach applied to any system, especially when using ABS to model complex adaptive systems (CAS) [33, $34]$. We validate the proposed simulation with the method mentioned in [35]. This part presents the operational validation of the simulation, and the empirical validation will be performed in the real case study.

5.4.1. Statistical Tests of Randomness Effects. In order to examine the extent to which the randomness affects the simulation results, experiment was repeated ten times. The results are 
TABLE 3: Strategy effectiveness results of successive events.

\begin{tabular}{lccccc}
\hline Criteria & $\begin{array}{c}\text { Without strategy } \\
\text { (single event) }\end{array}$ & Without strategy & Speakers controlling & Interaction probability & Communication intervention \\
\hline$F_{1}$ & 547 & $6465(+1081.90 \%)$ & $0(-100 \%)$ & $3236(-49.95 \%)$ & $5460(-15.55 \%)$ \\
$F_{2}$ & 8220 & $6185(-24.76 \%)$ & $0(-100 \%)$ & $5474(-11.50 \%)$ & $5918(-4.32 \%)$ \\
$F_{3}$ & 250.61 & $270.85(+8.08 \%)$ & $251.89(-7.00 \%)$ & $229.44(-15.29 \%)$ & $258.11(-4.7 \%)$ \\
$T_{1}$ & 131 & $190(+45.04 \%)$ & $0(-100 \%)$ & $187(-1.57 \%)$ & $189(-0.52 \%)$ \\
$T_{2}$ & 45 & $84(+86.67 \%)$ & $46(-45.24 \%)$ & $86(+2.38 \%)$ & $86(+2.38 \%)$ \\
$T_{3}$ & 17 & $18(+5.88 \%)$ & $0(-100 \%)$ & $33(+83.33 \%)$ & $18(0 \%)$ \\
$T_{4}$ & 45 & $124(+175.56 \%)$ & $0(-100 \%)$ & $86(-30.65 \%)$ & $95(-23.39 \%)$ \\
$T_{5}$ & 20 & $25(+25.00 \%)$ & $0(-100 \%)$ & $38(+52.00 \%)$ & $25(0 \%)$ \\
$T_{6}$ & 111 & $165(+48.65 \%)$ & $0(-100 \%)$ & $149(-9.70 \%)$ & $164(-0.61 \%)$ \\
\hline
\end{tabular}

TABLE 4: Statistical significance for the three strategies.

\begin{tabular}{lccc}
\hline Criteria & $\begin{array}{c}\text { Speakers } \\
\text { controlling }\end{array}$ & $\begin{array}{c}\text { Interaction } \\
\text { probability }\end{array}$ & $\begin{array}{c}\text { Communication } \\
\text { intervention }\end{array}$ \\
\hline$F_{1}$ & 0.608 & 0.000 & 0.000 \\
$F_{2}$ & 0.221 & 0.000 & 0.000 \\
$F_{3}$ & 0.152 & 0.000 & 0.000 \\
$T_{1}$ & 0.437 & 0.000 & 0.818 \\
$T_{2}$ & 0.407 & 0.000 & 0.169 \\
$T_{3}$ & 0.340 & 0.000 & 0.022 \\
$T_{4}$ & 0.849 & 0.000 & 0.166 \\
$T_{5}$ & 0.470 & 0.000 & 0.003 \\
$T_{6}$ & 0.411 & 0.000 & 0.872 \\
\hline
\end{tabular}

shown in Figure 6, and the statistical significance results are shown in Table $4(P<0.01)$. From Figure 6, we could find that the randomness extent is changing with the rate of variables changing, and this is due to the speed of information spreading. From the entire period, the randomness has no significance effect on the evolution process. From the results in Table 4, we could find that the interaction probability strategy significantly affects all the outcomes, and speakers controlling strategy has little effect on the single event. Communication strategy works well on the statistical group emotion but not on the event time, except the rise time period of agitated group.

5.4.2. Sensitivity Analysis. We then examined the effectiveness difference under different parameter values for sensitivity analysis, and the results are shown in Figure 7. As described in Sections 5.2 and 5.3, the effectiveness of speakers controlling strategy only depends on the execution time, while the other two depend on the probabilities. Thus, in this examination, we focus on the intervention strategy probability, that is, the interaction probability and link removing probability corresponding to the two strategies. From Figure 7, we could find that the probabilities have a significance effect on the effectiveness of the strategies. Besides, the closer the probability is to the critical value $\left(P_{\text {in }} \rightarrow 0, P_{\text {re }} \rightarrow 1\right)$, the more significant the effect is.
5.5. A Case Study. The computational model mentioned above was applied to the Urumqi incident in Xinjiang province (China). The incident took place on July 5, 2009, when approximately 20,000 people had ever received the violence activity information through all kinds of channels, and almost 5000 people attended the activities. The incident started as the information spreading of Shaoguan (Guangdong, China) incident among the population of Xinjiang by the terrorists organization Hizb ut-Tahrir [36] with extreme violence emotion instigation. More details of the incidents can be found in [4]. Based on the research method proposed, the effectiveness of intervention strategies was tested through the simulation experiments.

To simulate the incident, the events-chain model was constructed based on the events time during the incident, and meta-events are presented in Table 5. According to the incident evolution information, the meta-events were modeled as related information diffusion events during the process. The time of the events was translated into the simulation steps as events attributes. The criticalities of the events were given manually based on the instigation degree of the information, which were also mentioned in [4]. The agent number was 20000, and the other parameters were set as default values in Table 1 .

Figure 8 shows the average emotion evolution results during the incident, and derivative events are marked on the figure according to the event time. The blue events are the meta-events used in the events-chain model, and the red events are the derivative violence activities during the incident. It is easy to find that the derivative events usually occurred at the peak points of the collective emotions and had the highest participation at these points. Another interesting result is that the meta-events usually occurred at the concave points of the curve, which means that the violence activities information is propagated at the low points of the collective emotion curves.

The emotion evolution results and the collective derivative events (events 6, 7, 8) show good consistency in Figure 8. This indicates the validity of proposed method from the macro tendency perspective. As the detailed individual data is impossible to obtain, the macro emergent results proved that ABS provides a feasible way to study the collective emotion evolution in this type of incidents. Furthermore, the 


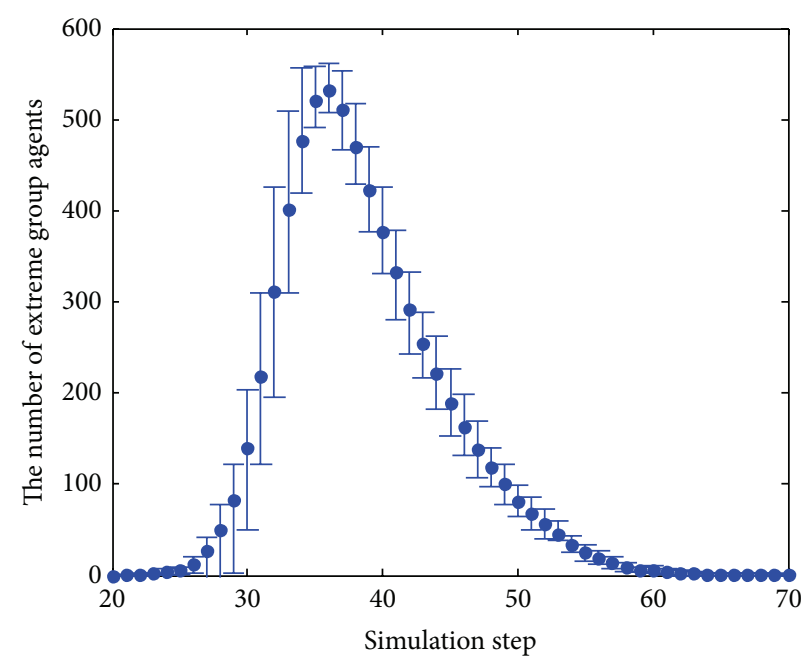

(a)

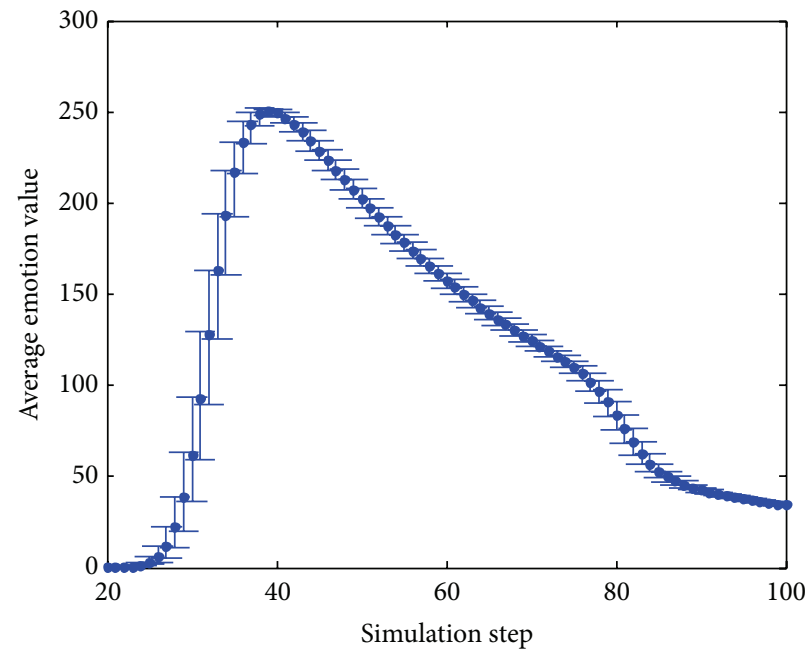

(c)

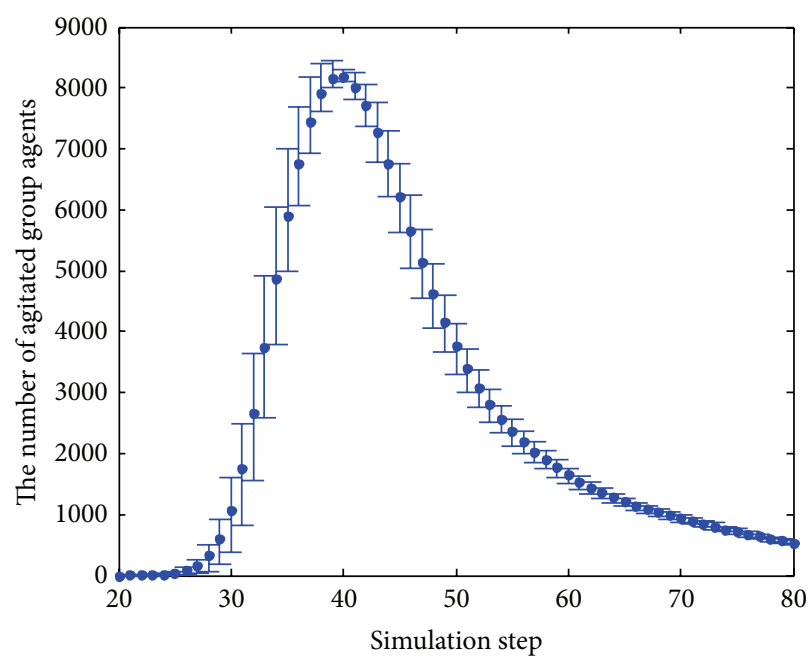

(b)

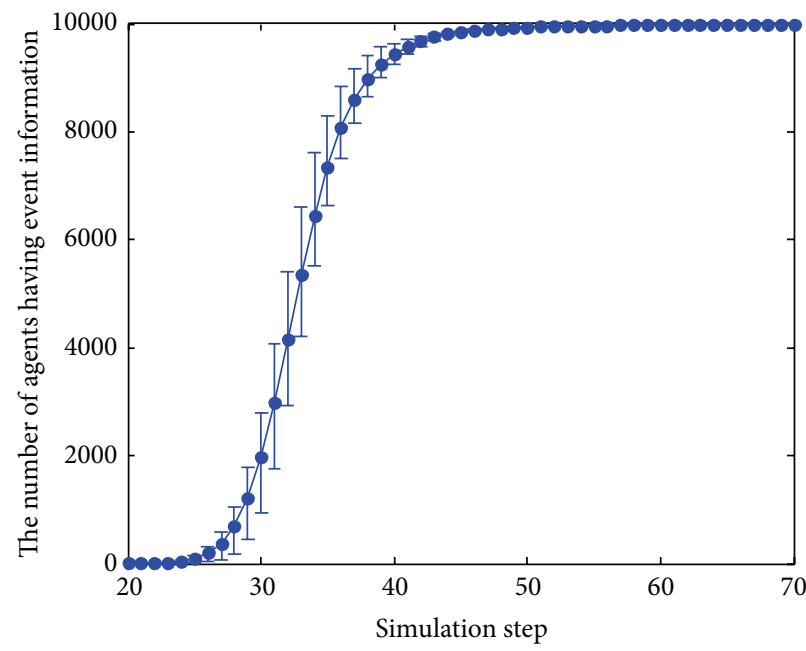

(d)

FIGURE 6: Randomness influence on the results (without strategy, single event).

TABLE 5: Meta-events of the case incident.

\begin{tabular}{lcccc}
\hline Event number & Event time & Simulation step & Criticality & Event \\
\hline 1 & June 25 & 125 & 1 & The Shaoguan incident \\
2 & June 27 & 175 & 3 & The statement of Shaoguan \\
3 & June 30 & 250 & 4 & Violence activity information \\
4 & July 2 & 300 & 2 & Activity information \\
5 & July 5 & 375 & 5 & Violence activity instigation \\
\hline
\end{tabular}

consistency between the events and group emotion can be used in events forecasting, which is also an important issue in emergency management.

To compare the simulation results with the real events for model validation, the derivative events are listed in Table 6. The time of derivative events is translated into simulation step. The start time of derivative violence events is obtained as the time at peak points. The number of extreme people is the number of extreme groups at the peak points. As a complex system simulation, the macro evolution tendency is much more important for understanding the emergent characteristics of the system. The consistency of the derivative events and simulation results also shows the validity of proposed method.

Figure 9 shows the influences of different strategies on the collective emotion evolution. The tendency of curves is similar to the results in Section 5.3, which indicates that the effectiveness of the strategies has no 


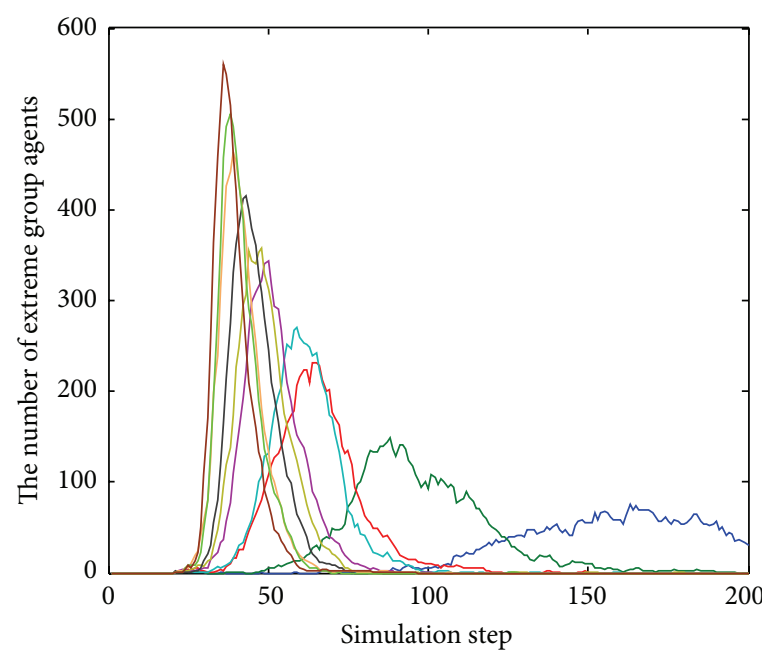

(a)

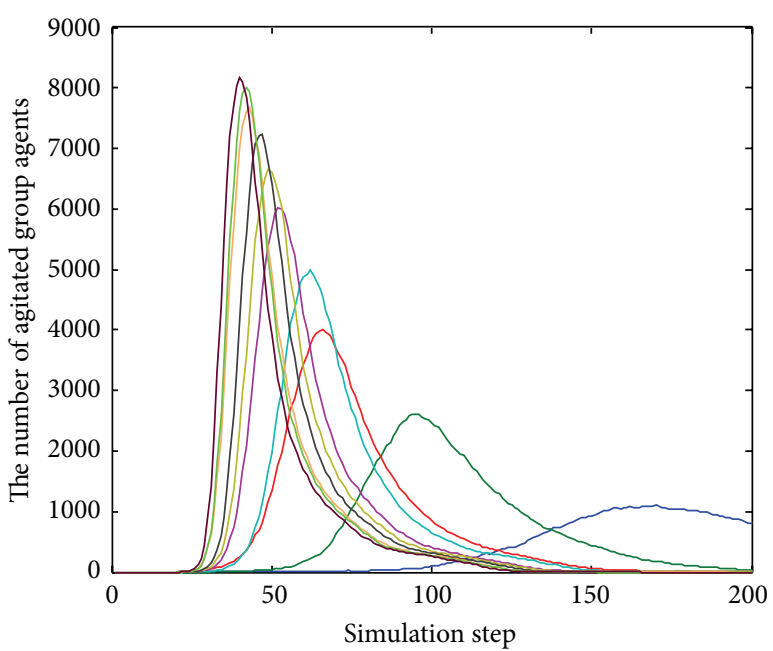

(c)

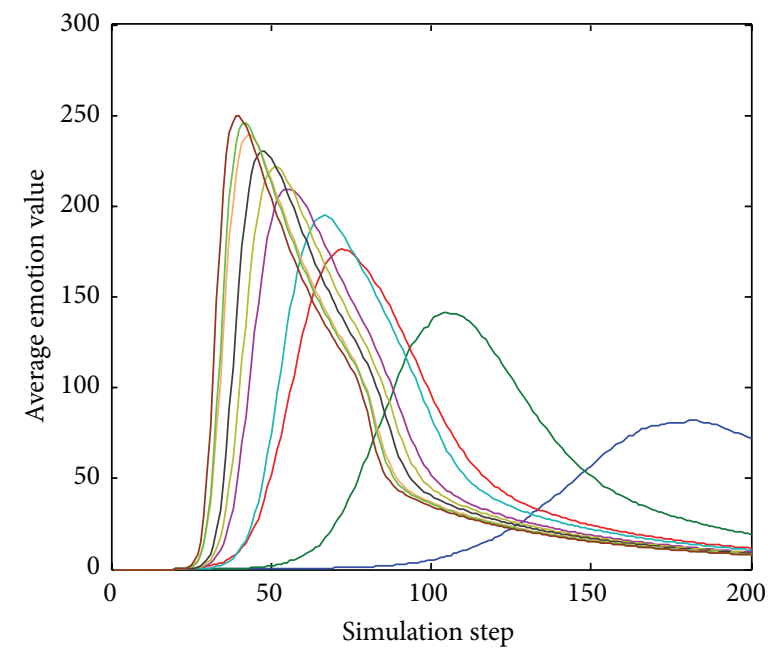

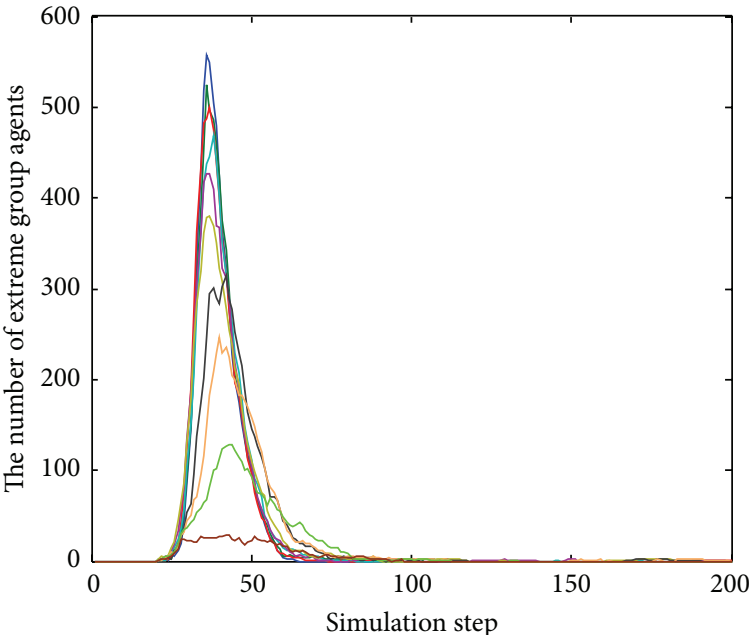

(b)

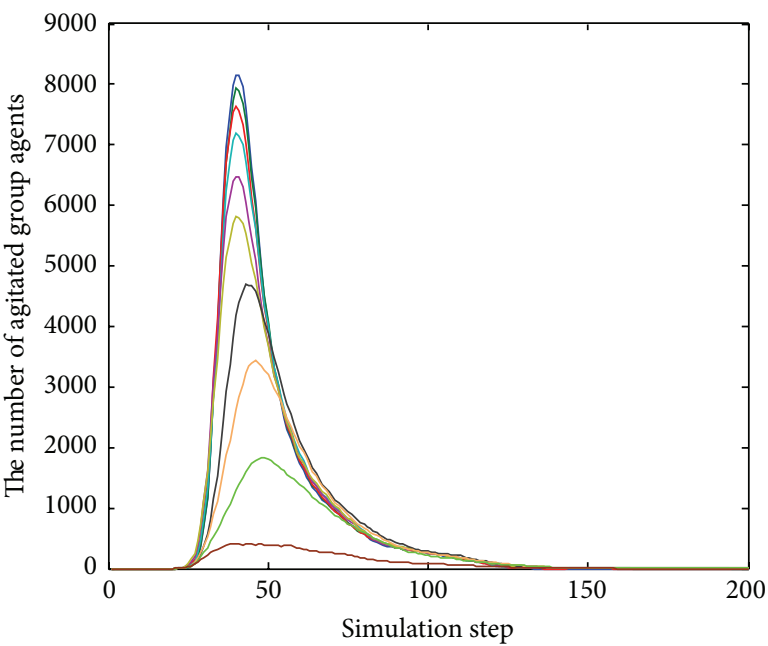

(d)

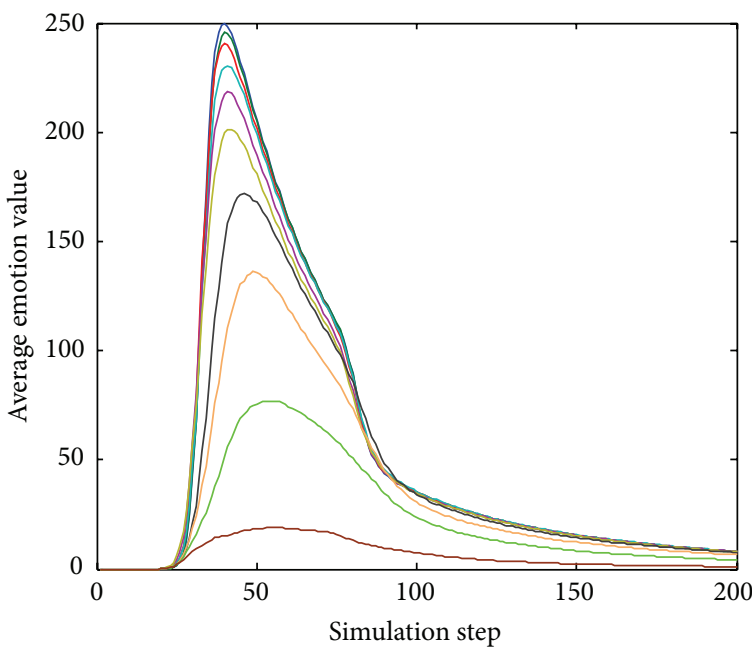

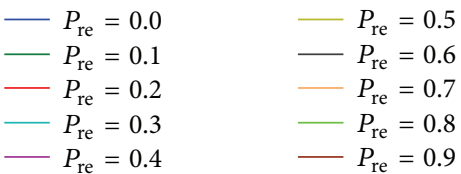

(f)

(e)

FIgURe 7: Continued. 


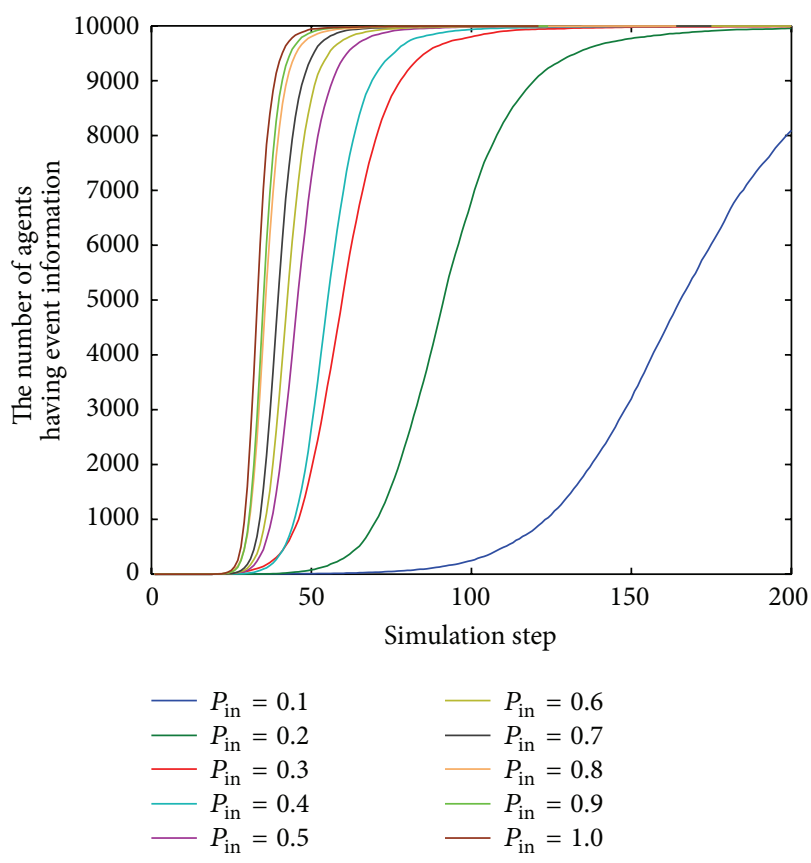

(g)

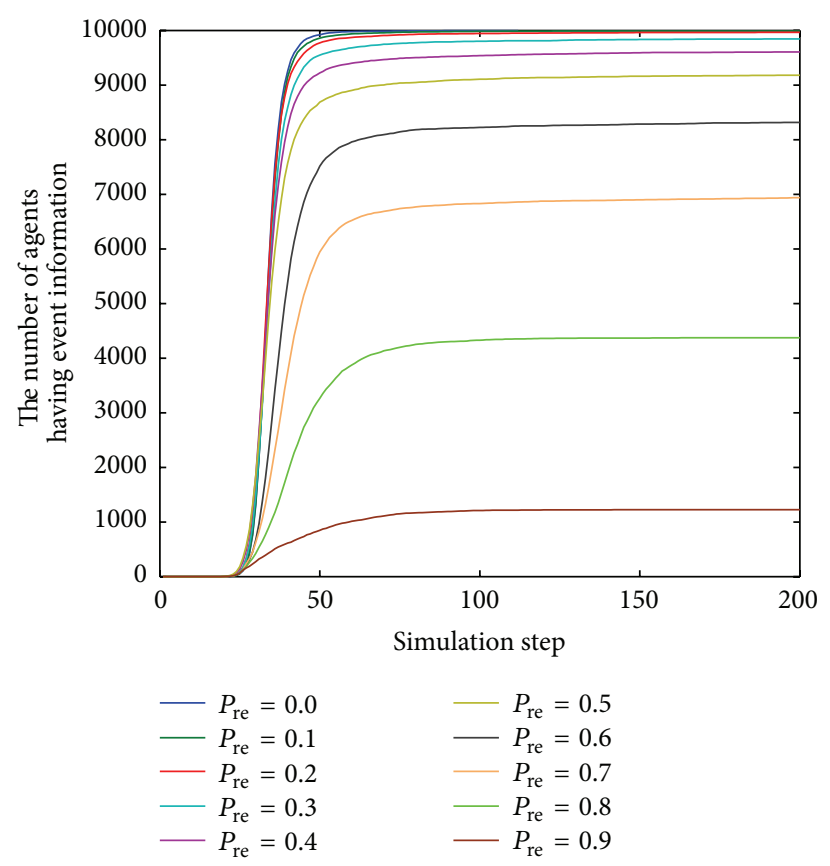

(h)

FiguRE 7: Parameters difference influence on the strategies effectiveness. (a), (c), (e), and (g) interaction probability strategy. (b), (d), (f), and (h) Communication intervention strategy.

TABLE 6: Derivative events in the case incident.

\begin{tabular}{lccccc}
\hline Number & Date & Simulation step & Experiments results & Extreme people (estimated) & Experiments results \\
\hline 6 & June 28 & 200 & 193 & 50 & 54 \\
7 & July 1 & 280 & 267 & 1800 & 1634 \\
8 & July 7 & 400 & 392 & 5000 & 5277 \\
\hline
\end{tabular}

TABLE 7: Strategy effectiveness results of the case incident.

\begin{tabular}{lcccc}
\hline Criteria & Without strategy & Speakers controlling & Interaction probability & Communication \\
\hline$F_{1}$ & 5277 & $30(-99.43 \%)$ & $3144(-40.42 \%)$ & $4629(-12.28 \%)$ \\
$F_{2}$ & 8924 & $8266(-7.37 \%)$ & $7763(-13.01 \%)$ & $8364(-6.28 \%)$ \\
$F_{3}$ & 263.57 & $158.43(-39.89 \%)$ & $227.27(-13.77 \%)$ & $250.45(-4.98 \%)$ \\
$T_{2}$ & 122 & $25(-79.51 \%)$ & $133(+9.02 \%)$ & $123(+0.82 \%)$ \\
\hline
\end{tabular}

obvious difference under different events simulation scenarios. The speakers controlling strategy is still the most effective method for reducing the emotion of successive events, and the interaction probability strategy can slow the emotion spread speed and reduce the number of group agents.

Table 7 shows experiment results of the incident simulation. In this experiment, since there is a series of events, the time characteristics of single event are the focus, except the "risk period" indicator $\left(T_{2}\right)$. Based on the "rise and fall" pattern of collective emotion [6], $T_{2}$ presents the total accumulation time when the average emotion is above the threshold of $\tau_{3}$. As the strategies were taken after the second event occurred (step 200), the speakers controlling strategy is still the most effective method for successive violence events. The interaction probability strategy is effective in reducing both group agents and average emotion value. The communication intervention strategy also has slight effect on the collective emotion evolution.

This case study simulates the group emotion evolution in the Urumqi incident and examines the effectiveness of the three strategies. The consistency shows that the proposed method is useful to describe the evolution process of this type of incidents, and the method of strategies evaluation provides 


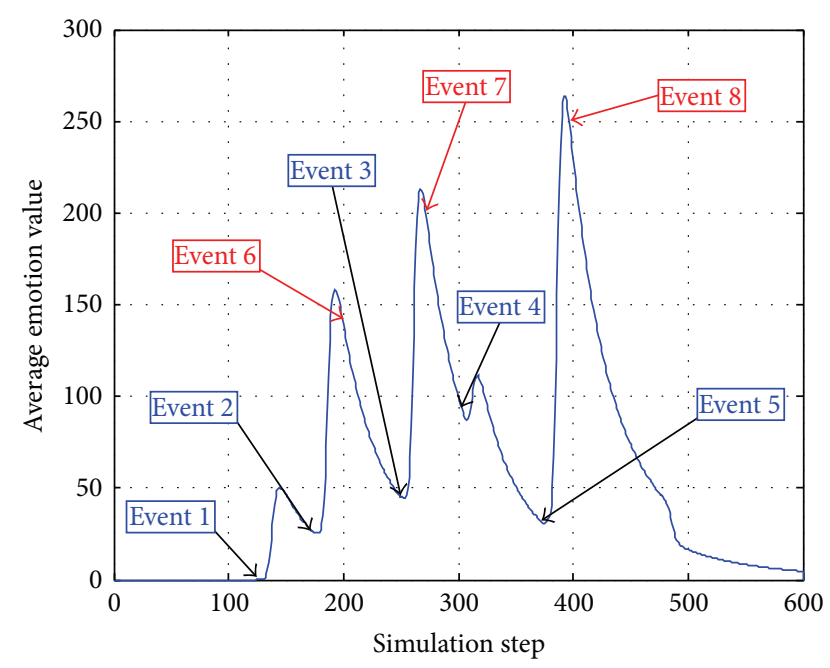

Event 1: The Shaoguan incident Event 2: The statement of Shaoguan Event 3: Violence activity information Event 4: Activity information Event 5: Violence activity instigation Event 6: Individual violence activities Event 7: Group violence activities Event 8: Large-scale violence activities

FIGURE 8: Group emotion evolution and events in the case incident.

a convenient tool for the strategy formulation to intervene in the incidents.

\section{Conclusion}

This paper studied the dynamics of the group emotion evolution and effectiveness of intervention strategies with ABS method in extreme events. Modeling and simulating the process of information spreading and emotion evolution provide some insight into potentially useful strategies. Through the use of ABS, the complexities of the collective emotion evolution and group behavior can be better understood. The strategies can be tested in simulation environment that behave similarly to real world situation. To further understand the accumulation influence of successive events, the events-chain model is proposed to model the continuous relevant events using simulation experiments. In this model, the continuous events are expressed as a series of metaevents and information spreading processes. The information diffusion between individuals is the driving power of emotion and behavior changing. In order to evaluate strategies' effectiveness, three strategies are modeled based on mathematical definitions. Nine criteria are defined from the statistical and temporal perspectives, which measure the collective motion dynamics with statistical properties and evolution curve characteristics. Based on the constructed simulation system, three experiments are performed as well as model validation. The simulation results allow us to derive several conclusions, either on the collective emotion evolution or on the strategies effectiveness.
Our main conclusions are summarized as follows.

(i) The group extreme emotion evolution process is the collective response dynamics of events, accompanying information diffusion. The dynamics generate wave pattern with the increase of time, which can be regarded as pulses of events.

(ii) The environmental influence emotion is the main cause that makes individual's emotion become extreme. The extreme emotion infection between agents generates centralized emotion distribution, which shows convergence in collective emotion dynamics.

(iii) For single event, interaction probability is the most effective strategy, either in reducing the number of group agents or in slowing the evolutionary process. Communication can only reduce the peak value of the group agents, and speakers controlling has little effectiveness if it is taken after the event starts.

(iv) For successive events, because of the accumulation influence, the speakers controlling is the most useful strategy for preventing the derivative events, as it removes the information source of the events. The interaction probability strategy displays similar results in single event, as well as the communication intervention strategy.

(v) The sensitive analysis indicates that the probabilities are the deterministic factor that determines the effectiveness of speakers controlling and communication intervention strategies. Meanwhile, the statistical test of randomness effects proves that the model is robust and valid.

Moreover, the real case study shows consistency between the group emotion dynamics and the collective behavior (violence activities events). This makes it possible to predict group emotion evolution tendency and test effectiveness of various strategies. The predictive value would be significant for government in managing and preventing extreme incidents.

In conclusion, the presented agent based simulations give a new insight into group extreme emotion dynamics in extreme events. It can be used to test variety of simulation experiments for understanding the emotion dynamics associated with other models. Meanwhile, based on the simulation framework, other intervention strategies can be evaluated through corresponding models. Hence, despite its mathematical complexity, our method provides a laboratory for further experiments on the group emotional behavior of agents (e.g., under different driving conditions, varied agent behaviors, and parameters values) and for comparative analysis of intervention strategies. A future development of the research would be to apply more models of sophisticated events, including events classification (distinguishing different types of events and agents' response to them) and events impact model (how the events influence the agents' behavior). Furthermore, heterogeneity of agents also should be considered in the simulation. 


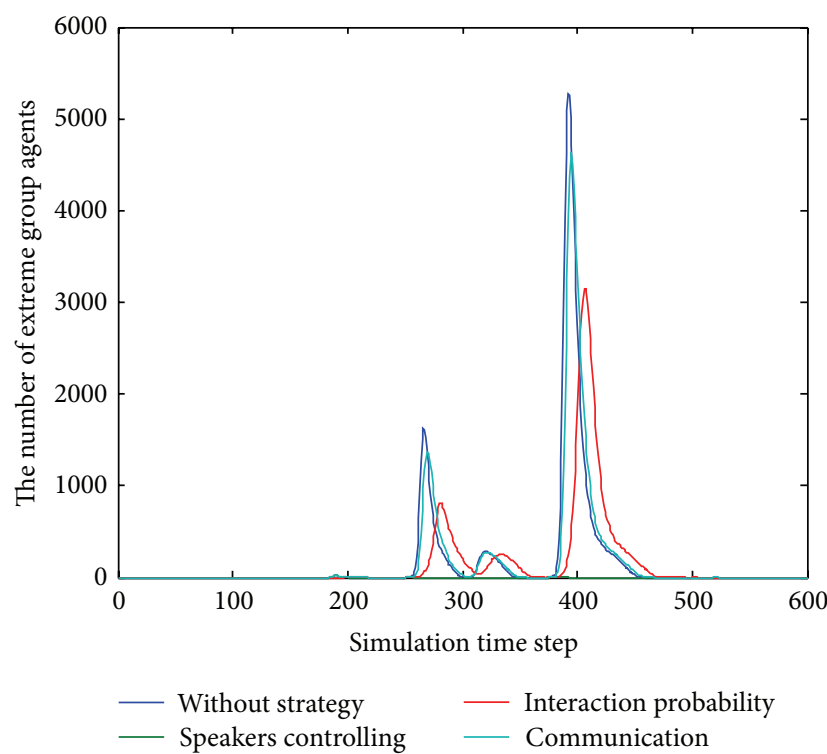

(a)

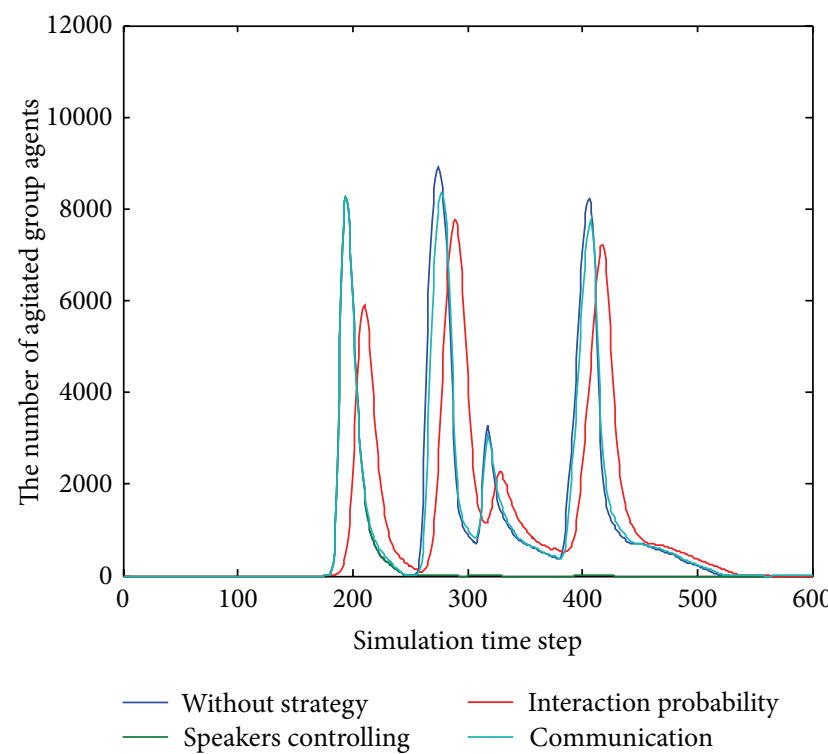

(b)

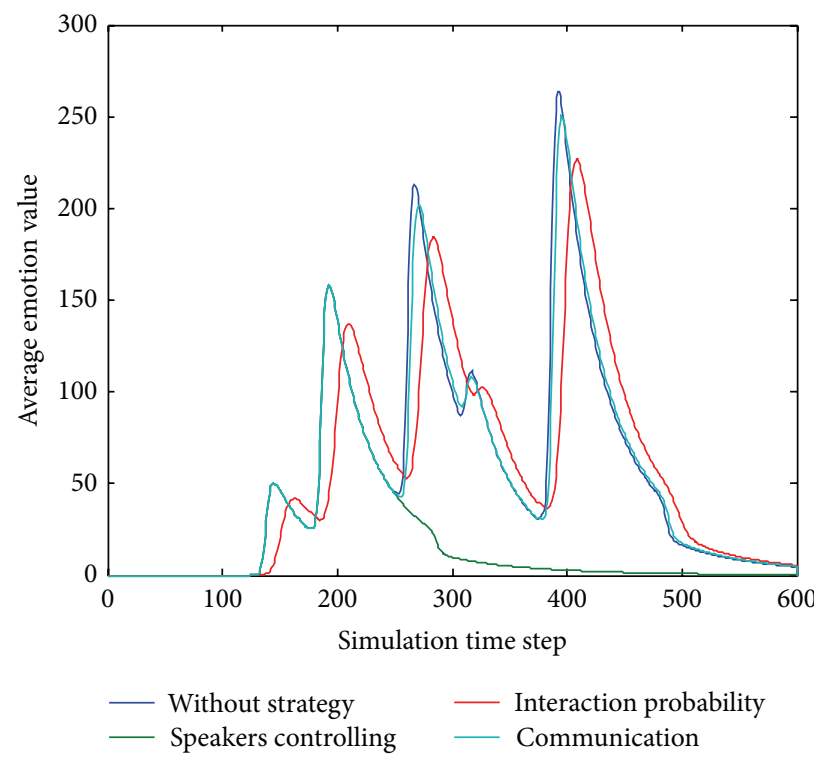

(c)

FIGURE 9: Group emotion evolution of the case incident, (a) the number curves of extreme group agents, (b) the number curves of agitated group agent, and (c) average emotion value evolution curves. (The strategies were taken at simulation step 200.)

\section{Conflict of Interests}

The authors declare that there is no conflict of interests regarding the publication of this paper.

\section{Acknowledgments}

This work is supported by the National Natural Science Foundation of China (Grant no. 71473263) and the Specialized Research Fund for the Doctoral Program of Higher Education of China (Grant no. 20134307110020).

\section{References}

[1] I. J. Roseman, M. S. Spindel, and P. E. Jose, "Appraisals of emotion-eliciting events: testing a theory of discrete emotions," Journal of Personality and Social Psychology, vol. 59, no. 5, pp. 899-915, 1990.

[2] D. Bar-Tal, E. Halperin, and J. de Rivera, "Collective emotions in conflict situations: societal implications," Journal of Social Issues, vol. 63, no. 2, pp. 441-460, 2007.

[3] A. Wedeman, "Enemies of the state: mass incidents and subversion in China," in Proceedings of the Annual Meeting of the American Political Seience Association, Toronto, Canada, 2009. 
[4] WebPage, http://en.wikipedia.org/wiki/July_2009_Urumqi_riots.

[5] M. Nekovee, Y. Moreno, G. Bianconi, and M. Marsili, “Theory of rumour spreading in complex social networks," Physica A: Statistical Mechanics and Its Applications, vol. 374, no. 1, pp. 457470, 2007.

[6] Y. Matsubara, Y. Sakurai, B. A. Prakash, L. Li, and C. Faloutsos, "Rise and fall patterns of information diffusion: model and implications," in Proceedings of the 18th ACM SIGKDD International Conference on Knowledge Discovery and Data Mining (KDD '12), pp. 6-14, Beijing, China, August 2012.

[7] T. Bosse, M. Hoogendoorn, M. C. A. Klein, J. Treur, C. N. van der Wal, and A. van Wissen, "Modelling collective decision making in groups and crowds: integrating social contagion and interacting emotions, beliefs and intentions," Autonomous Agents and Multi-Agent Systems, vol. 27, no. 1, pp. 52-84, 2013.

[8] R. L. Goldstone and M. A. Janssen, "Computational models of collective behavior," Trends in Cognitive Sciences, vol. 9, no. 9, pp. 424-430, 2005.

[9] Y. Sano, K. Yamada, H. Watanabe, H. Takayasu, and M. Takayasu, "Empirical analysis of collective human behavior for extraordinary events in the blogosphere," Physical Review E, vol. 87, no. 1, Article ID 012805, 2013.

[10] T. Bosse, M. Hoogendoorn, M. Klein et al., "Agent-based modelling of social emotional decision making in emergency situations," in Co-Evolution of Intelligent Socio-Technical Systems, pp. 79-117, Springer, Berlin, Germany, 2013.

[11] J. Duffy, "Agent-based models and human subject experiments," in Handbook of Computational Economics, L. Tesfatsion and K. L. Judd, Eds., pp. 950-1011, Elsevier, 2006.

[12] J. H. Miller and S. E. Page, Somplex Adaptive Systems: An Introduction to Computational Models of Social Life, Princeton University Press, 2009.

[13] C. M. MacAl and M. J. North, "Tutorial on agent-based modelling and simulation," Journal of Simulation, vol. 4, no. 3, pp. 151-162, 2010.

[14] S. J. Alam and A. Geller, "Networks in agent-based social simulation," in Agent-Based Models of Geographical Systems, A. J. Heppenstall, Ed., Springer Science+Business Media B.V., 2012.

[15] I.-C. Moon and K. M. Carley, "Modeling and simulating terrorist networks in social and geospatial dimensions," IEEE Intelligent Systems, vol. 22, no. 5, pp. 40-49, 2007.

[16] R. Colbaugh and K. Glass, "Early warning analysis for social diffusion events," Security Informatics, vol. 1, no. 18, pp. 1-26, 2012.

[17] F. Bergenti, E. Franchi, and A. Poggi, "Agent-based interpretations of classic network models," Computational and Mathematical Organization Theory, vol. 19, no. 2, pp. 105-127, 2013.

[18] J. P. Keller, K. C. Desouza, and Y. Lin, “Dismantling terrorist networks: evaluating strategic options using agent-based modeling," Technological Forecasting \& Social Change, vol. 77, no. 7, pp. 1014-1036, 2010.

[19] D. M. Mackie, T. Devos, and E. R. Smith, "Intergroup emotions: explaining offensive action tendencies in an intergroup context," Journal of Personality and Social Psychology, vol. 79, no. 4, pp. 602-616, 2000.

[20] T. A. B. Snijders, C. E. G. Steglich, and M. Schweinberger, "Modeling the co-evolution of networks and behavior," in Longitudinal Models in the Behavioral and Related Sciences, $\mathrm{K}$. V. Montfort, J. Oud, and A. Satorra, Eds., pp. 41-71, Erlbaum Associates Publishers, 2007.
[21] A. Apolloni, K. Channakeshava, L. Durbeck et al., "A study of information diffusion over a realistic social network model," in Proceedings of the IEEE International Conference on Computational Science and Engineering (CSE '09), vol. 4, pp. 675-682, Vancouver, Canada, August 2009.

[22] A. L. Barabasi and R. Albert, "Emergence of scaling in random networks," Science, vol. 286, no. 5439, pp. 509-512, 1999.

[23] S. Field, "Self-determination instructional strategies for youth with learning disabilities," Journal of Learning Disabilities, vol. 29, no. 1, pp. 40-52, 1996.

[24] K. A. Maria and R. A. Zitar, "Emotional agents: a modeling and an application," Information and Software Technology, vol. 49, no. 7, pp. 695-716, 2007.

[25] G. Marreiros, R. Santos, C. Ramos, and J. Neves, "Contextaware emotion-based model for group decision making," IEEE Intelligent Systems, vol. 25, no. 2, pp. 31-39, 2010.

[26] R. Axelrod, The Complexity of Cooperation: Agent-Based Models of Competition and Collaboration, Princeton University Press, Princeton, NJ, USA, 1997.

[27] T. W. Valente, "Network interventions," Science, vol. 336, no. 6090, pp. 49-53, 2012.

[28] S. P. Borgatti, "Identifying sets of key players in a social network," Computational and Mathematical Organization Theory, vol. 12, no. 1, pp. 21-34, 2006.

[29] T. Bosse, R. Duell, Z. A. Memon, J. Treur, and C. N. van der Wal, "A multi-agent model for mutual absorption of emotions," in Proceedings of the 23rd European Conference on Modelling and Simulation (ECMS '09), pp. 212-218, June 2009.

[30] A. B. Krueger and J. Malečková, "Attitudes and action: public opinion and the occurrence of international terrorism," Science, vol. 325, no. 5947, pp. 1534-1536, 2009.

[31] D. Kempe, J. Kleinberg, and E. Tardos, "Influential nodes in a diffusion model for social networks," in Automata, Languages and Programming, vol. 3580 of Lecture Notes in Computer Science, pp. 1127-1138, Springer, Berlin, Germany, 2005.

[32] N. Gilbert, Agent Based Models, Sage, 2007.

[33] S. Moss, "Alternative approaches to the empirical validation of agent-based models," Journal of Artificial Societies and Social Simulation, vol. 11, no. 1, p. 15, 2008.

[34] L. Yilmaz, "Validation and verification of social processes within agent-based computational organization models," Computational and Mathematical Organization Theory, vol. 12, no. 4, pp. 283-312, 2006.

[35] P. Windrum, G. Fagiolo, and A. Moneta, "Empirical validation of agent-based models: alternatives and prospects," The Journal of Artificial Societies and Social Simulation, vol. 10, no. 2, pp. 119, 2007.

[36] http://en.wikipedia.org/wiki/Hizb_ut-Tahrir. 


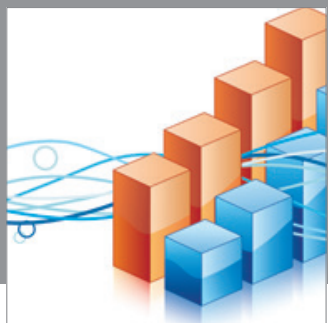

Advances in

Operations Research

mansans

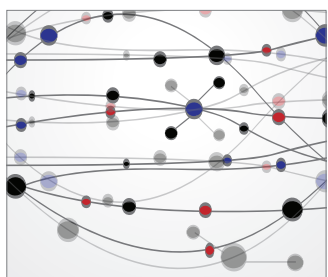

The Scientific World Journal
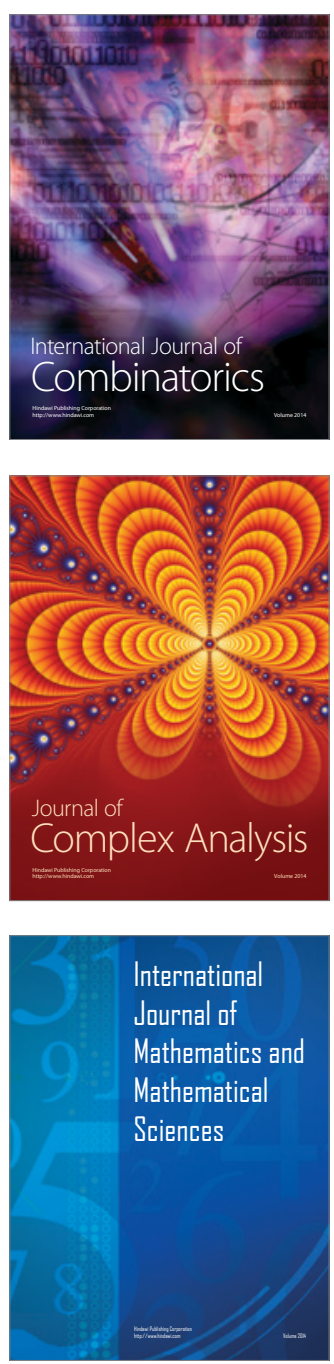
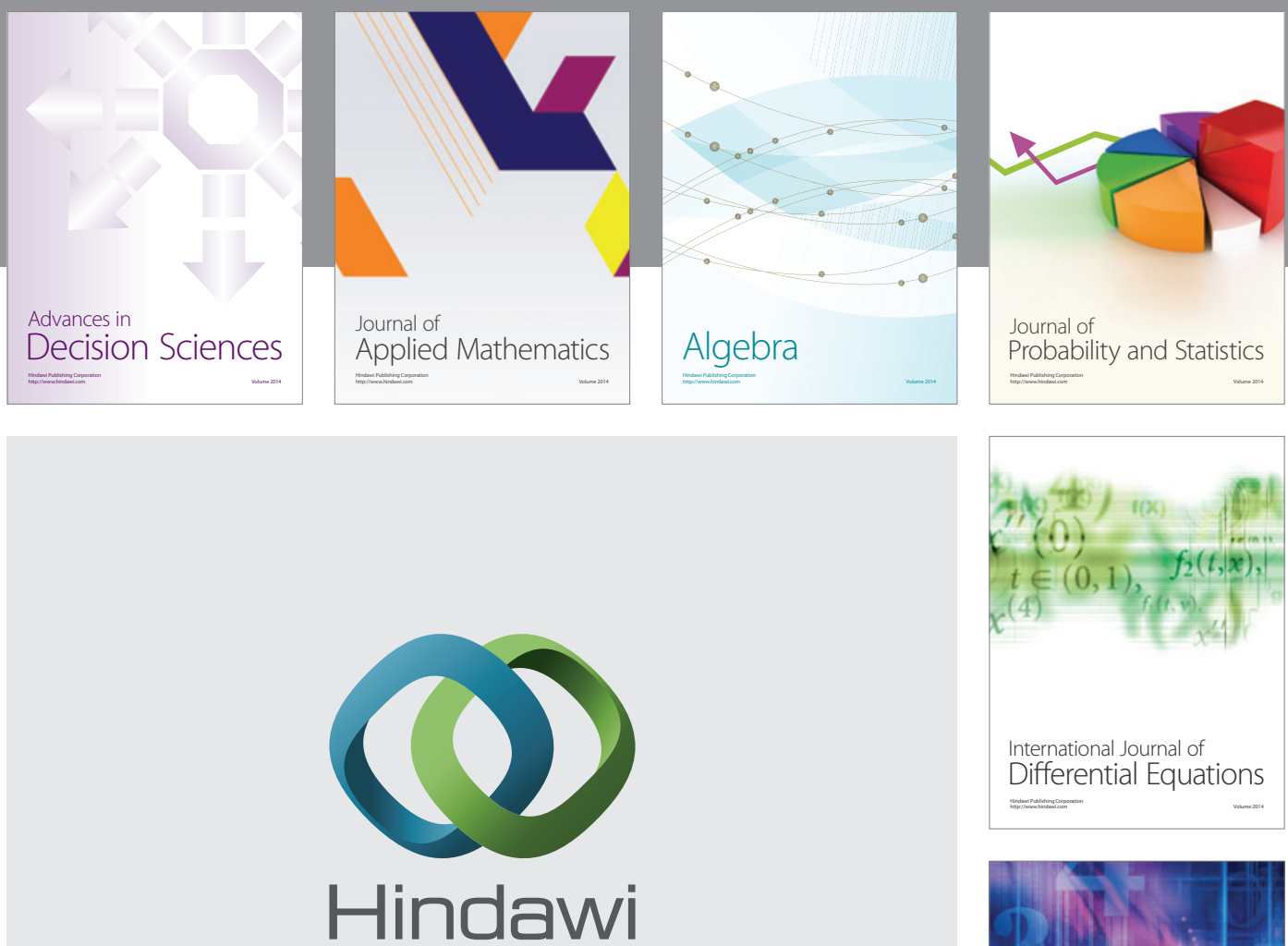

Submit your manuscripts at http://www.hindawi.com
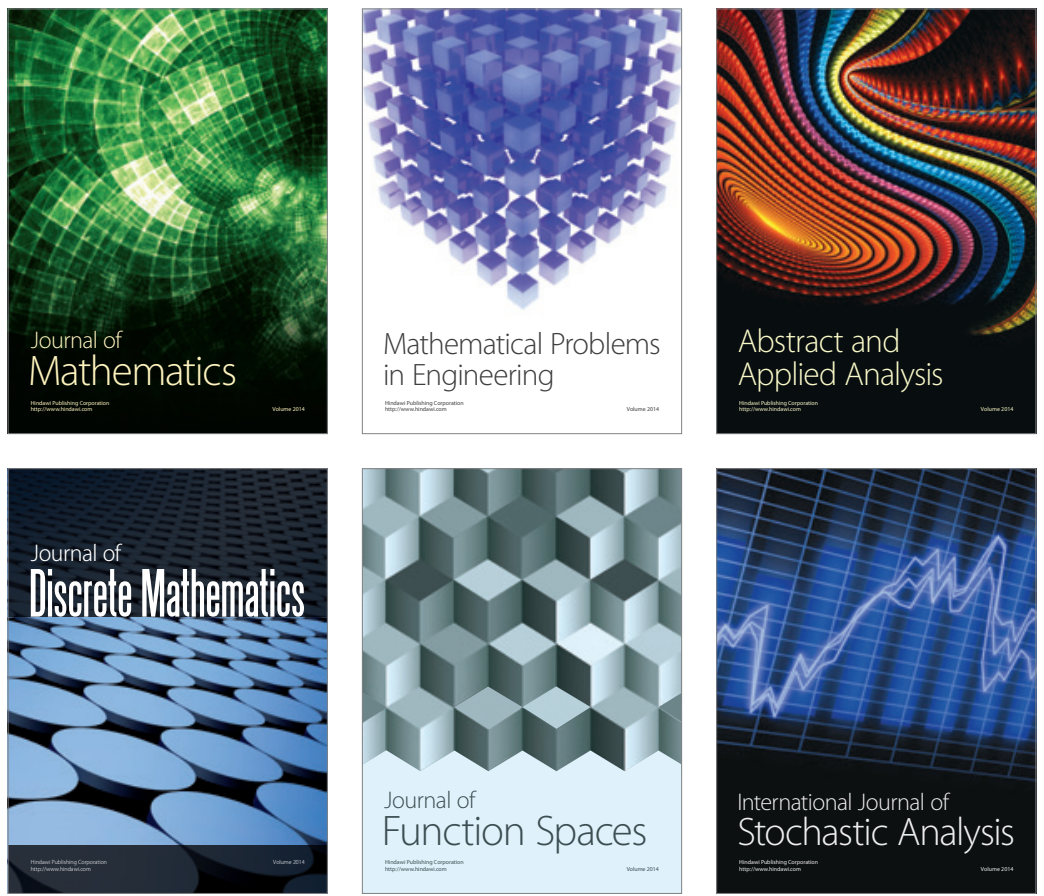

Journal of

Function Spaces

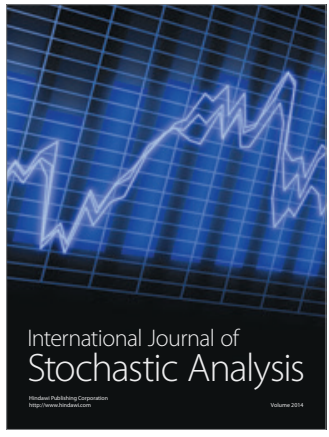

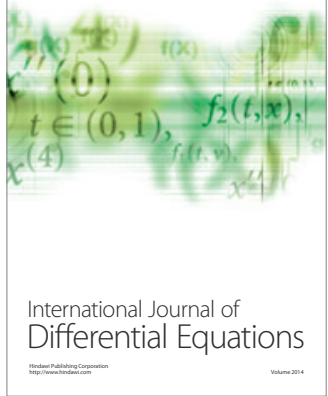
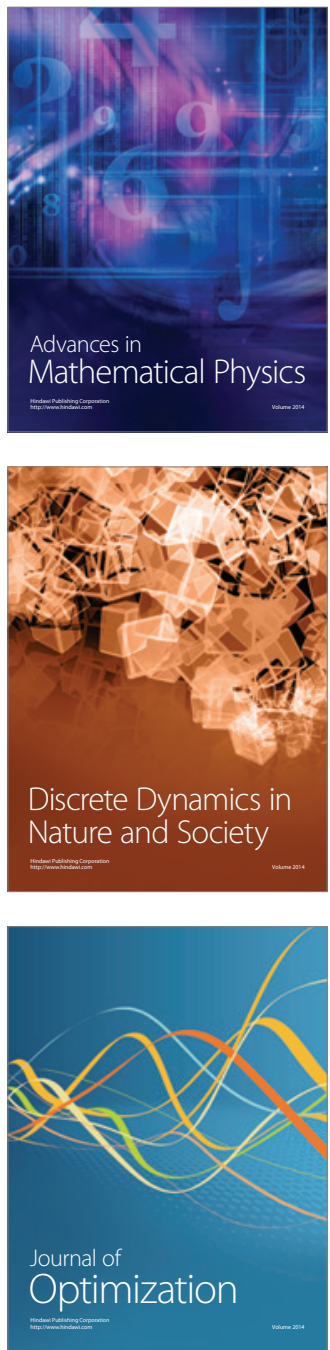